\title{
A Novel Speckle Noise Removal Algorithm Based on ADMM and Energy Minimization Method
}

\author{
Bo Chen $\mathbb{D}^{1,2}$ Yan Lv, ${ }^{1}$ Jinbin Zou, ${ }^{1}$ Wensheng Chen, ${ }^{1,2}$ and Binbin Pan $\mathbb{D}^{1,2}$ \\ ${ }^{1}$ Shenzhen Key Laboratory of Advanced Machine Learning and Applications, College of Mathematics and Statistics, \\ Shenzhen University, Shenzhen 518060, China \\ ${ }^{2}$ Guangdong Key Laboratory of Intelligent Information Processing, Shenzhen University, Shenzhen 518060, China
}

Correspondence should be addressed to Bo Chen; chenbo@szu.edu.cn

Received 14 June 2020; Accepted 20 July 2020; Published 12 August 2020

Academic Editor: Xinguang Zhang

Copyright ( 2020 Bo Chen et al. This is an open access article distributed under the Creative Commons Attribution License, which permits unrestricted use, distribution, and reproduction in any medium, provided the original work is properly cited.

Speckle noise removal in medical ultrasound images is a challenging task. In this paper, a new model is proposed to removal speckle noise, alternating direction method of multipliers algorithm is employed to solve the new energy minimization model. The convexity, existence, and uniqueness of the new energy minimization model's solution are proved. Series of experiments are designed in this paper. Numerical results show that the new algorithm can reduce the step effect effectively obtain good results in visual effect and quantitative measures by comparing with some traditional models.

\section{Introduction}

With the rapid development of science and technology, computer network and image equipment are widely used. There are more and more application fields of digital image, including pattern recognition, medical imaging, video processing, remote sensing, and other fields. At the same time, people have higher and higher requirements for the quality of digital image, and the digital image processing technology has attracted more and more attention of researchers.

Image denoising technology is mainly to input a degraded image with noise into the computer for processing, to eliminate the noise in the image, make it closer to the original image, and facilitate the subsequent processing. In the early image denoising work, it can be divided into filter based on convolution methods $[1,2]$, wavelet based on image denoising methods [3, 4], depth based on learning method, and partial differential equation (PDE) based methods [5-8].

Medical ultrasound image reflects the difference of acoustic parameters in media and can obtain information different from optical, X-ray and Y-ray. Ultrasound has a good ability to distinguish human soft tissues, which is helpful to identify micropathological changes in biological tis- sues. But in the process of medical ultrasound image transmission, speckle noise will be produced, which will lead to a significant decline in the quality of ultrasound image and cover up the damage of some important tissues. How to eliminate speckle noise in ultrasound image while retaining its important features is a challenge.

In Ref. [9], Loupas et al. proved by experiments that the noise in medical ultrasound image is no longer the multiplicative noise in the form of Rayleigh distribution, and also proved in the paper that the form of medical ultrasound degraded image can be written:

$$
f=u+\sqrt{u} n,
$$

where $f$ is a noisy image and $n$ represents the Gaussian random noise with zeros mean and standard deviation $\sigma$.

In the paper [2], Kristian et al. derive the corresponding data fidelity term according to the distribution characteristics of Gaussian noise and the degradation model of medical ultrasonic noise image:

$$
\int_{\Omega} \frac{(f-u)^{2}}{u} d x
$$




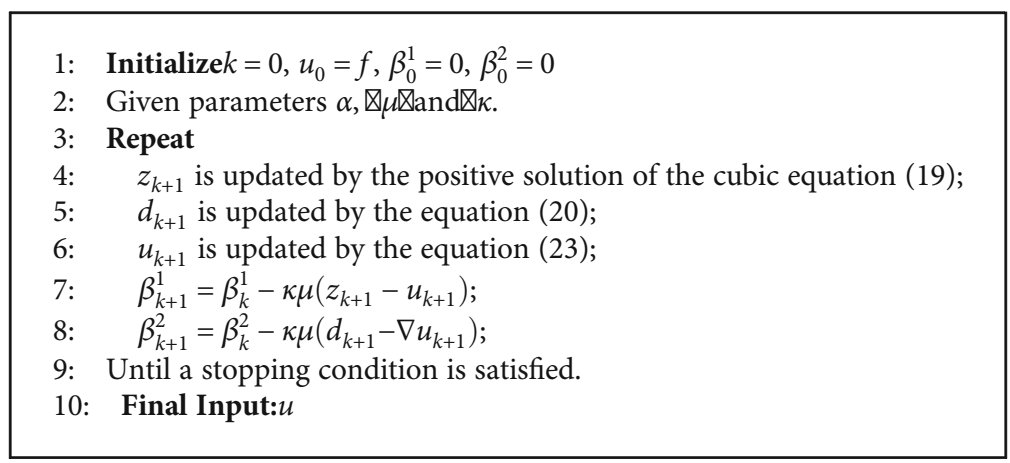

Algorithm 1: ADMM algorithm for the new model.

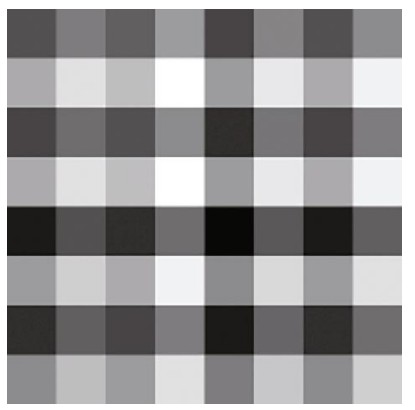

(a)

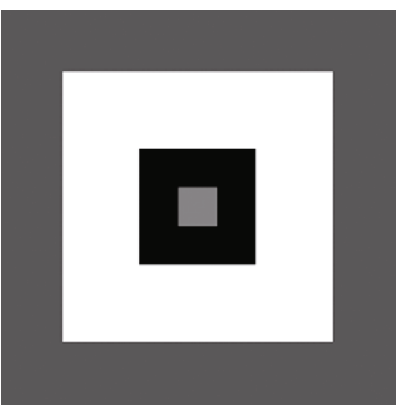

(b)

Figure 1: Original images. (a) syn $1(256 \times 256)$. (b) syn $2(512 \times 512)$.

With the passage of time, mathematical research is constantly improving, so stochastic theory [10-14] and PDE [15-20] have been fully developed, and Fractional theory [21, 22], wavelet [23], and statistical information [24] are all employed to deal with intensity inhomogeneity or noise. In this paper, the method of partial differential equation is used to solve speckle noise. In the numerical algorithm, although the use of image denoising is effective, it will not only bring some ladder effect but also low iterative efficiency. Therefore, the optimization algorithm has received great attention in recent years. Many efficient optimization algorithms have been proposed, such as Split Bregman type iterative [25], the Douglas-Rachford method [26], primal-dual algorithm [27], and Alternating Direction Method of Multipliers algorithm (ADMM) [26, 28]. In this paper, we will use the ADMM algorithm to solve the new energy minimization model.

The rest of this paper is as follows. In Section 2, we introduce the new energy minimization model and the ADMM algorithm. In Section 3, we adopt the ADMM algorithm to solve the new energy minimization model. Section 4 shows some numerical results and compares them with other existing models. The conclusion is drawn in Section 5.

\section{Background}

2.1. The TV Model. In 1992, the total variation (TV) model was proposed, which is the classical total variational model.
The forms of TV model are generally as follows:

$$
\min \int_{\Omega}|\nabla u| d x+\frac{\lambda}{2} \int_{\Omega}\left|u-u_{0}\right|^{2} d x
$$

where $u: \Omega \rightarrow R$ is a clean image and $u_{0}$ is a noisy image affected by Gaussian random noise with mean zero and standard deviation $\sigma . \lambda>0$ is regularization parameter which can balance fidelity term and regularized term in TV model. $\nabla$ represents the gradient operator, which is defined as follows:

$$
\nabla u=\left(\partial_{x}^{+} u, \partial_{y}^{+} u\right)
$$

where $\partial_{x}^{+}, \partial_{y}^{+}$is the forward discrete derivation operators, which are defined as:

$$
\begin{aligned}
& \partial_{x}^{+} u= \begin{cases}u_{i, j+1}-u_{i, j} & \text { if } 1 \leq j \leq N-1 \\
0 & \text { if } j=N\end{cases} \\
& \partial_{y}^{+} u= \begin{cases}u_{i+1, j}-u_{i, j} & \text { if } 1 \leq i \leq M-1 \\
0 & \text { if } i=M\end{cases}
\end{aligned},
$$

2.2. The New Energy Minimization Model. The new energy minimization model is an adaptive total variation model, 


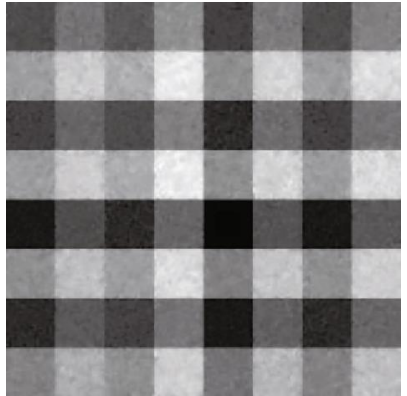

(a)

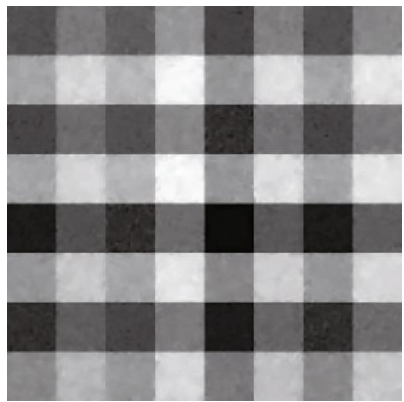

(c)

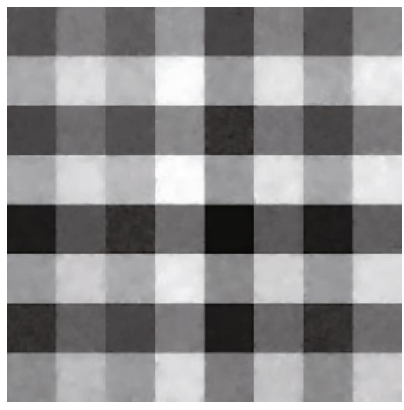

(e)

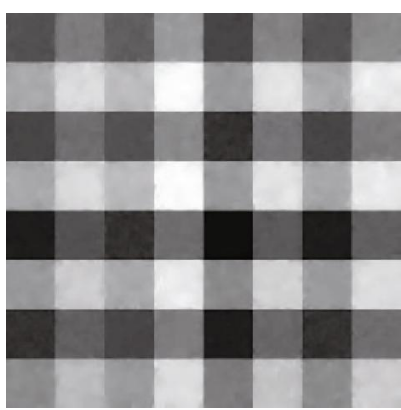

(g)

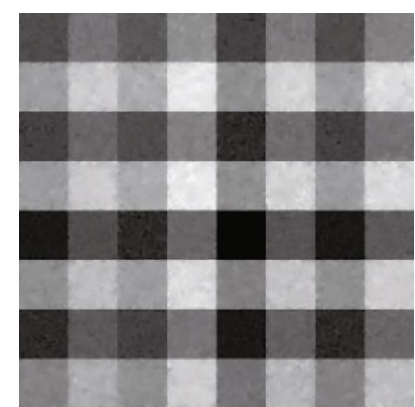

(b)

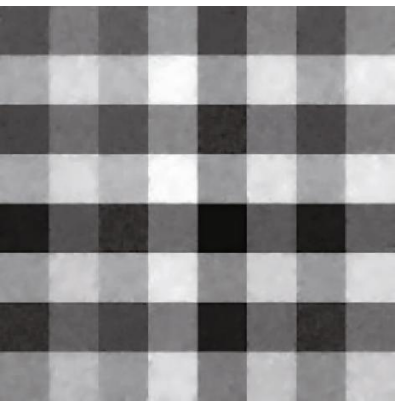

(d)

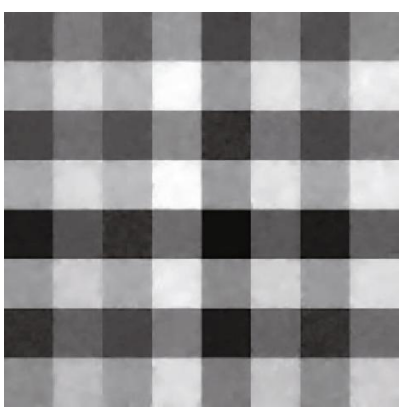

(f)

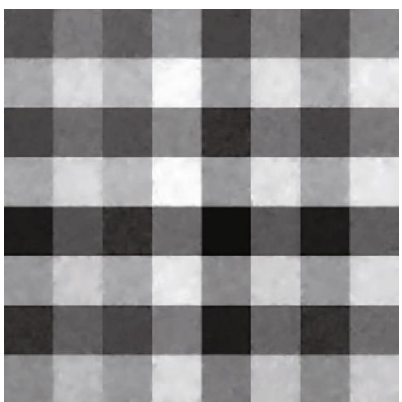

(h)

FIGURE 2: Continued. 


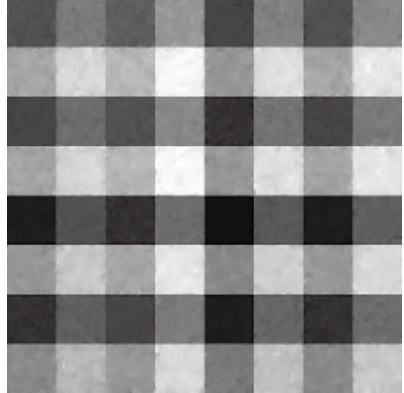

(i)

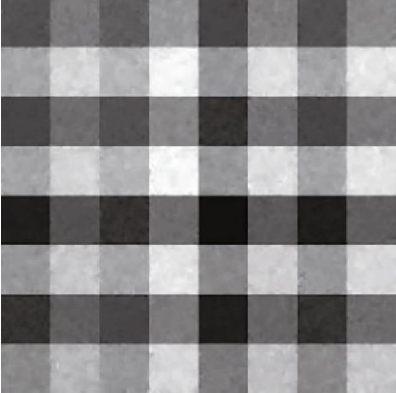

(j)

Figure 2: Numerical experiments with different parameters $\delta$ with noise standard deviation $\sigma=2$. (a) Restored image by $\delta=1$. (b) Restored image by $\delta=5$. (c) Restored image by $\delta=10$. (d) Restored image by $\delta=20$. (e) Restored image by $\delta=30$. (f) Restored image by $\delta=40$. (g) Restored image by $\delta=50$. (h) Restored image by $\delta=60$. (i) Restored image by $\delta=100$. (j)Restored image by $\delta=200$.
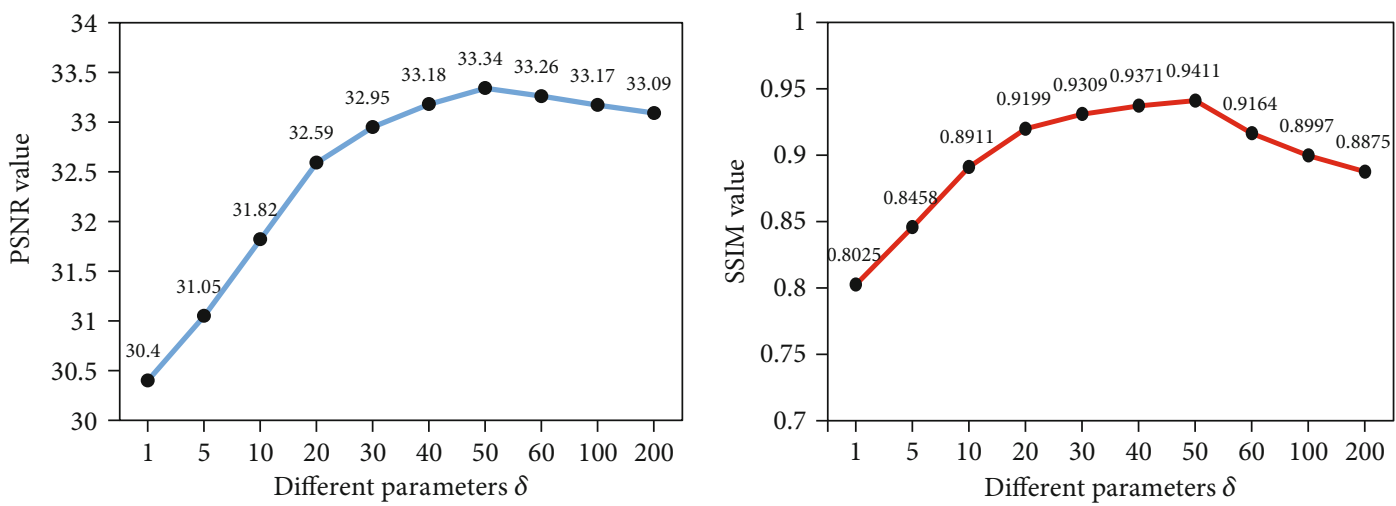

FIGURE 3: The PSNR and SSIM values of different $\delta$.

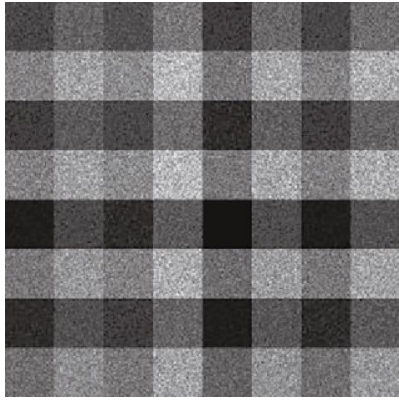

(a)

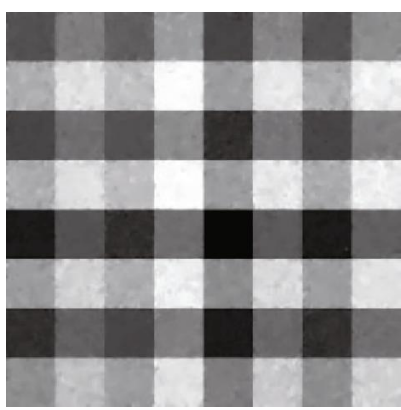

(c)

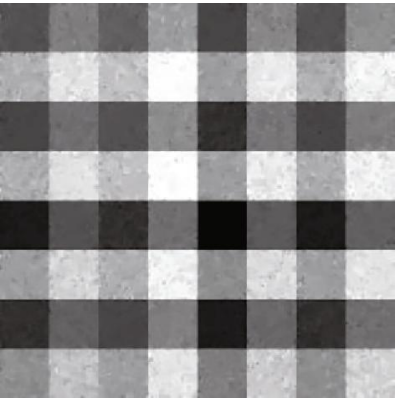

(b)

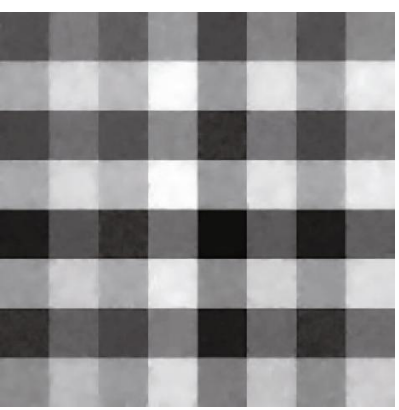

(d)

FIGURE 4: Numerical result of the "syn1" image with noise standard deviation $\sigma=2$. (a) Noisy image. (b) Restored image by the JIN's model. (c) Restored image by finite difference for the new model; (d) Restored image by ADMM for the new model. 


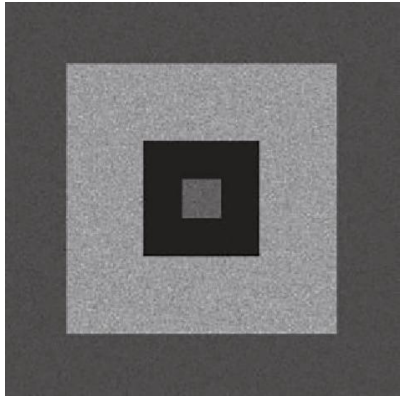

(a)

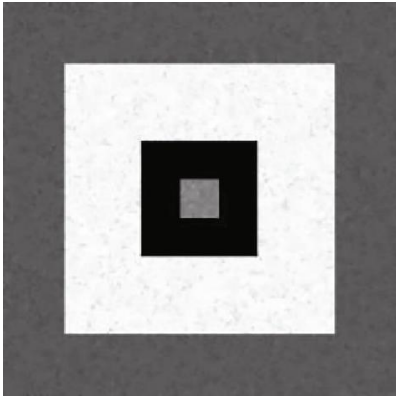

(b)

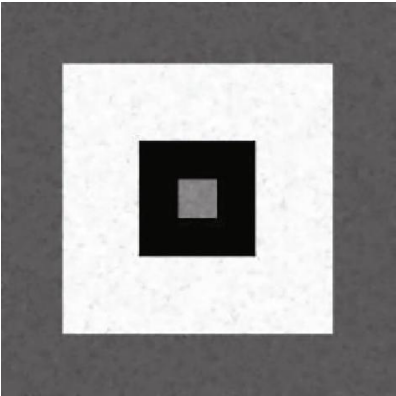

(c)

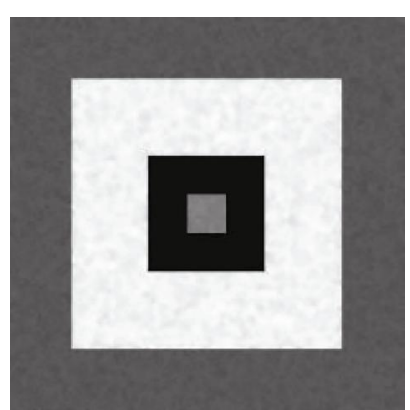

(d)

Figure 5: Numerical result of the "syn2" image with noise standard deviation $\sigma=3$. (a) Noisy image. (b) Restored image by the JIN's model. (c) Restored image by finite difference for the new model. (d) Restored image by ADMM for the new model.

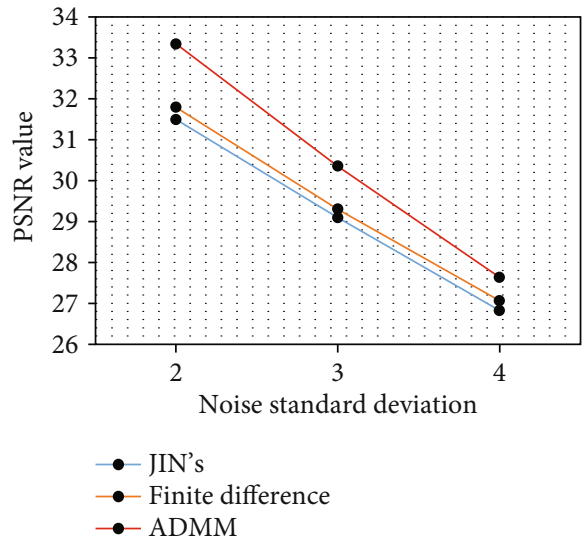

(a)

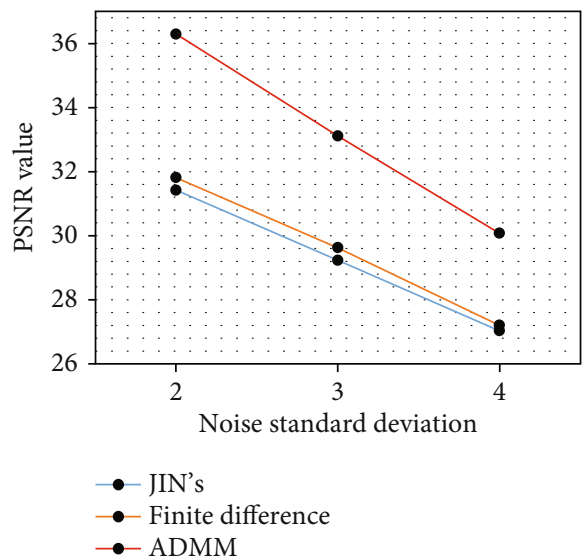

(c)

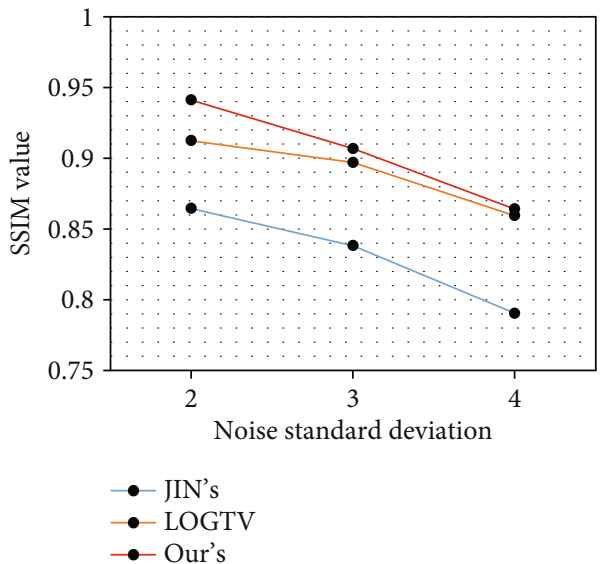

(b)

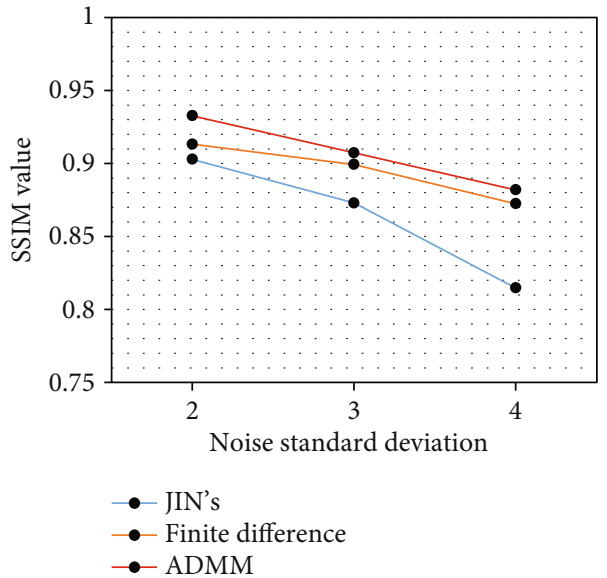

(d)

FIGURE 6: Folded-line contrast map. (a, b) PSNR and SSIM values of different models with different noise standard deviations ("syn1" image). (c, d) PSNR and SSIM values of different models with different noise standard deviations ("syn2" image).

which is defined by a new regularization term and the variance of speckle noise, as follows:

$$
\min _{u}\left[\int_{\Omega} \varphi(|\nabla u|) d x+\frac{\alpha}{2} \int_{\Omega} \frac{(f-u)^{2}}{u} d x\right],
$$

Where $\phi(s)=s \ln (\delta+s), \quad \int_{\Omega}\left((f-u)^{2} / u\right) \mathrm{d} x \quad$ is $\quad$ the fidelity term, and $\alpha$ represents the regularization parameter which can balance fidelity term and regularization term.

Firstly, the energy model (6) is convex, which guarantees the existence of the minimal solution of the model (6). 


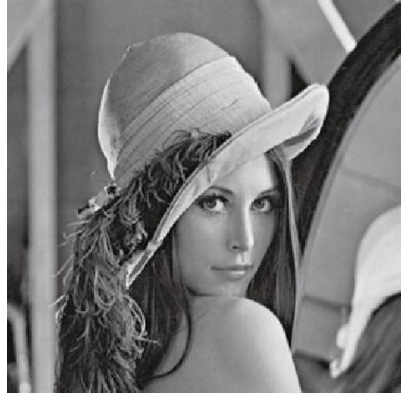

(a)

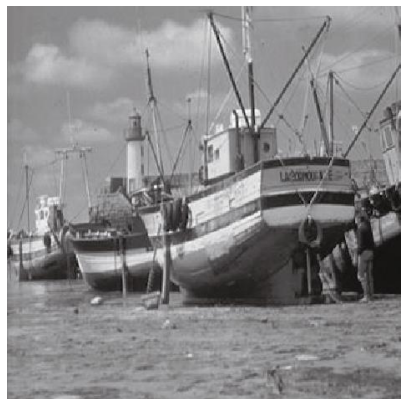

(c)

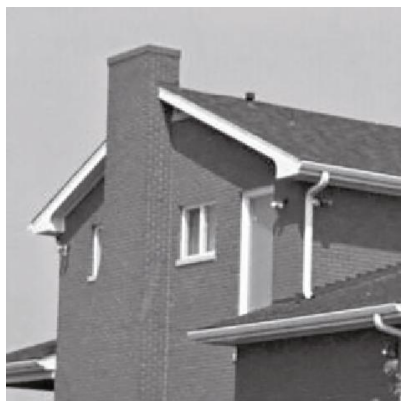

(e)

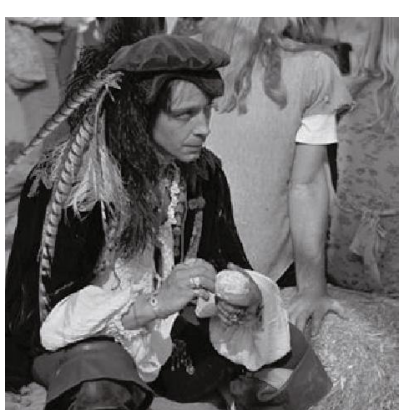

(b)

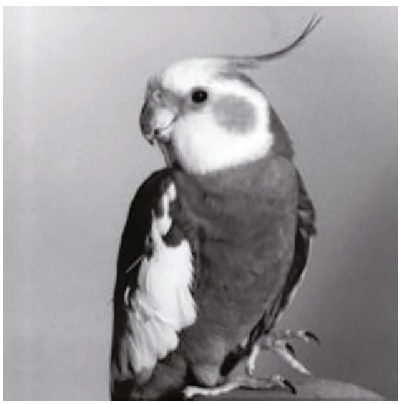

(d)

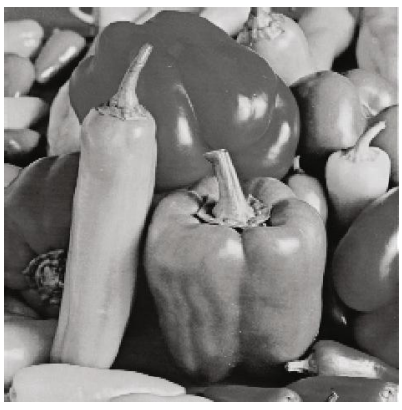

(f)

Figure 7: Original images: (a) lena $(512 \times 512)$; (b) pirate $(512 \times 512)$; (c) boat $(512 \times 512)$; (d) bird $(256 \times 256)$; (e) house $(256 \times 256)$; (f) peppers $(256 \times 256)$.

Theorem 1. The energy functional $E(u)$ is convex. That is to say, for any $u_{1}, u_{2} \in \Omega$ and $t \in[0,1]$, we have:

$$
E\left(t u_{1}+(1-t) u_{2}\right) \leq t E\left(u_{1}\right)+(1-t) E\left(u_{2}\right),
$$

where $E(u)=\int_{\Omega} \varphi(|\nabla u|) d x+(\alpha / 2) \int_{\Omega}\left((f-u)^{2} / u\right) d x$.

Proof. The proof of Theorem 1 is given in the appendix A.

Secondly, the uniqueness of the minimum solution of the model (6) can also be proved.

Theorem 2. If $u_{1}$ and $u_{2}$ are two minimize solutions of model (6), then we have $u_{1}=u_{2}$.

Proof. The proof of Theorem 2 is given in the appendix B.

2.3. Alternating Direction Method of Multipliers. Alternating Direction Method of Multipliers is a computational framework for solving optimization problems, which is suitable for solving distributed convex optimization problems.
ADMM decomposes a large global problem into several smaller, easy-to-solve local problems, and then solves them alternately. Its essence is the further development of the augmented Lagrange algorithm.

For linear constrained minimization problem,

$$
\min _{x, y} f(x)+g(y) \text { such that } A x+B y=b,
$$

where $x \in R^{n}, y \in R^{m}, A \in R^{k \times n}, B \in R^{k \times m}, b \in R^{k}$, and function $f: R^{n} \longrightarrow R$ and function $g: R^{m} \longrightarrow R$ are convex and lower semicontinuous functions.

By introducing a Lagrangian multiplier vector or dual variable $\beta \in R^{k}$, then the augmented Lagrange function of problem (8) is

$$
\begin{aligned}
L_{\mu}(x, y, \beta ; \mu)= & f(x)+g(y)-\beta^{T}(A x+B y-b) \\
& +\frac{\mu}{2}\|A x+B y-b\|_{2}^{2}
\end{aligned}
$$


where $\mu>0$ is a penalty parameter. By the algorithm of Alternating Direction Method of Multipliers (ADMM), the solution $\left(x^{k+1}, y^{k+1}\right)$ is achieved with the following iteration:

$$
\left\{\begin{array}{l}
x^{k+1}=\underset{x}{\arg \min } L_{\mu}\left(x, y^{k}, \beta^{k} ; \mu\right) \\
y^{k+1}=\underset{y}{\arg \min } L_{\mu}\left(x^{k+1}, y, \beta^{k} ; \mu\right) \\
\beta^{k+1}=\beta^{k}-\kappa \mu\left(A x^{k+1}+B y^{k+1}-b\right)
\end{array}\right.
$$

where $\sigma \in(0,(1+\sqrt{5} / 2))$ is a positive parameter. The convergence of the ADMM algorithm was proved in [29].

\section{The ADMM Algorithm for the Energy Minimization Model}

In this subsection, we will describe the ADMM algorithm for solving new energy minimization model. Firstly, the model (6) can be transformed into the following discretized version:

$$
\min _{u}\left[\langle\phi(|\nabla u|), \quad 1\rangle+\frac{\alpha}{2}\left\langle\frac{(f-u)^{2}}{u}, 1\right\rangle\right]
$$

To solve the problem with the ADMM algorithm, we introduce two new variables: $z, d=\left(d_{1}, d_{2}\right)$. Apply these two variables, the unconstrained problem (11) is written as the following constrained problem:

$$
\begin{gathered}
\min _{u}\left[\langle\phi(|d|), \quad 1\rangle+\frac{\alpha}{2}\left\langle\frac{(f-z)^{2}}{z}, 1\right\rangle\right], \\
\text { subject that } z=u, d=\nabla u
\end{gathered}
$$

Next, we make

$$
\begin{aligned}
& A=\left[\begin{array}{ll}
I & 0 \\
0 & I
\end{array}\right], x=\left[\begin{array}{l}
z \\
d
\end{array}\right] \\
& B=\left[\begin{array}{c}
-I \\
-\nabla
\end{array}\right], y=[u], \quad \beta=\left[\begin{array}{l}
\beta^{1} \\
\beta^{2}
\end{array}\right],
\end{aligned}
$$

Now, we letting

$$
f(x)=\langle\phi(|d|), 1\rangle+\frac{\alpha}{2}\left\langle\frac{(f-z)^{2}}{z}, 1\right\rangle \text { and } g(y)=0,
$$

So according to constraint problem (8), the constrained problem (12) can be solved by the ADMM algorithm. The augmented Lagrange function of problems (9) is

$$
\begin{aligned}
L_{\mu}\left(z, d, u, \beta^{1}, \beta^{2} ; \mu\right)= & \langle\phi(|d|), 1\rangle+\frac{\alpha}{2}\left\langle\frac{(f-z)^{2}}{z}, 1\right\rangle \\
& -\left(\beta^{1}\right)^{T}(z-u)-\left(\beta^{2}\right)^{T}(d-\nabla u) \\
& +\frac{\mu}{2}\|z-u\|_{2}^{2}+\frac{\mu}{2}\|d-\nabla u\|_{2}^{2},
\end{aligned}
$$

According to the structure of the ADMM algorithm, the problem (15) can be rewritten as the following form:

$$
\left\{\begin{array}{l}
\left(z_{k+1}, d_{k+1}\right)=\underset{z \geq 0, d}{\arg \min } L_{\mu}\left(z, d, u_{k}, \beta_{k}^{1}, \beta_{k}^{2} ; \mu\right) \\
u_{k+1}=\underset{d}{\arg \min } L_{\mu}\left(z_{k+1}, d_{k+1}, u, \beta_{k}^{1}, \beta_{k}^{2} ; \mu\right) \\
\beta_{k+1}^{1}=\beta_{k}^{1}-\kappa \mu\left(z_{k+1}-u_{k+1}\right) \\
\beta_{k+1}^{2}=\beta_{k}^{2}-\kappa \mu\left(d_{k+1}-\nabla u_{k+1}\right)
\end{array},\right.
$$

Since the two new variables $z$ and $d$ in problem (16) are not related to each other, so we can divide two simple minimization subproblem as follows,

$$
\left\{\begin{array}{l}
z_{k+1}=\min _{z \geq 0}\left[\frac{\alpha}{2}\left\langle\frac{(f-z)^{2}}{z}, 1\right\rangle-\left(\beta_{k}^{1}\right)^{T} z+\frac{\mu}{2}\left\|z-u_{k}\right\|_{2}^{2}\right], \\
d_{k+1}=\min _{d}\left[\left\langle\phi_{i}(|d|), 1\right\rangle-\left(\beta_{k}^{2}\right)^{T} d+\frac{\mu}{2}\left\|d-\nabla u_{k}\right\|_{2}^{2}\right]
\end{array}\right.
$$

First, for the $z$-subproblem, we can obtain the corresponding Euler-Lagrange equation:

$$
\frac{\alpha}{2}\left(1-\frac{f^{2}}{z^{2}}\right)-\beta_{k}^{1}+\mu\left(z-u_{k}\right)=0
$$

Simplifying the Euler-Lagrange equation above, we can obtain three complicated solutions by the roots formula of the cubic equation:

$$
2 \mu z^{3}+\left(\alpha-2 \beta_{k}^{1}-2 \mu u_{k}\right) z^{2}-\alpha f^{2}=0
$$

For equation (19), there is only one positive real number solution (the proof is given in appendix C). 


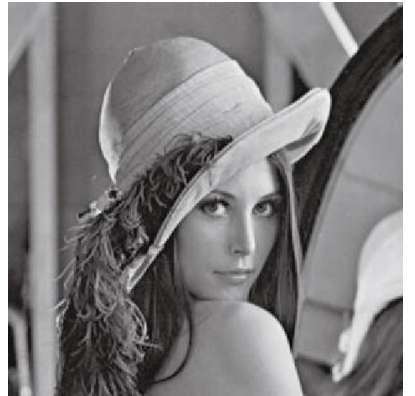

(a)

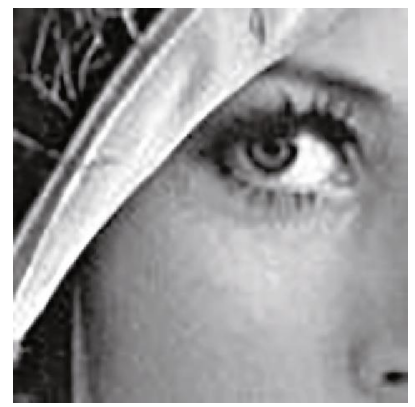

(e)

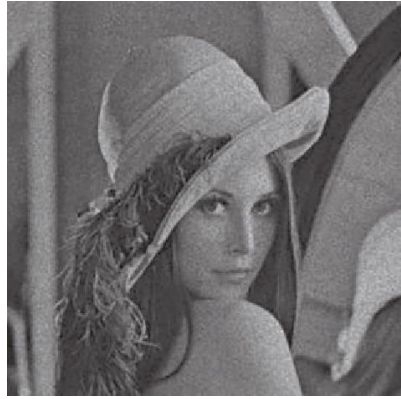

(b)

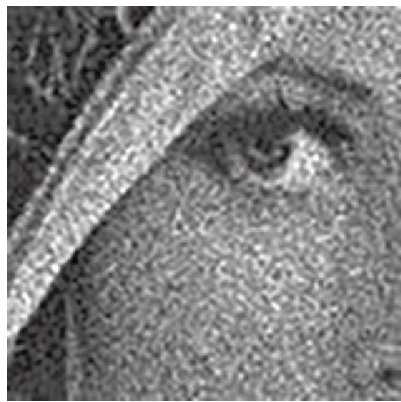

(f)

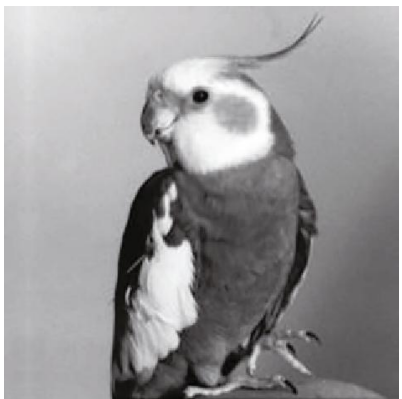

(c)

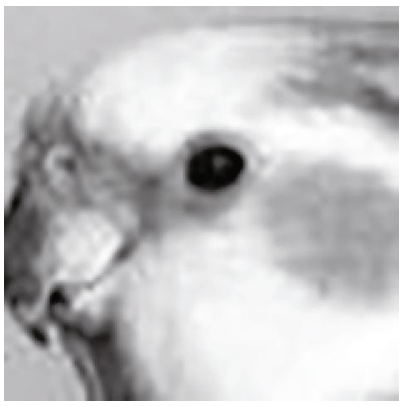

(g)

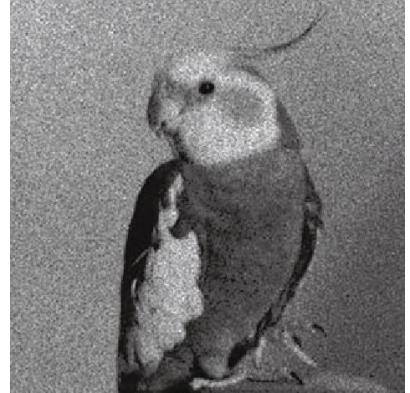

(d)

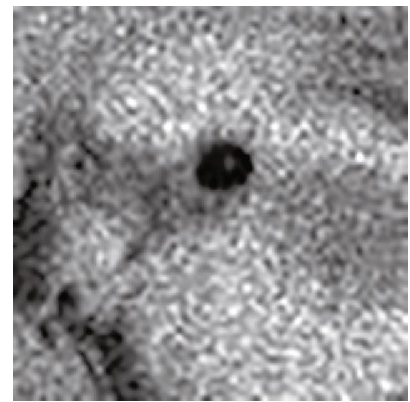

(h)

Figure 8: Numerical result of the "lena" and "bird" images with noise standard deviation $\sigma=2$. (a, c) Original images. (b, d) Noisy images. (eh) About the detailed image of $(a-d)$.

Second, for the $d$-subproblem, we will apply the shrink operator to obtain the solution $d$. The form of the solution $d$ is as follows:

$$
\begin{aligned}
d_{k+1} & =\min _{d}\left[\left\langle\phi_{i}(|d|), 1\right\rangle-\left(\beta_{k}^{2}\right)^{T} d+\frac{\mu}{2}\left\|d-\nabla u_{k}\right\|_{2}^{2}\right] \\
& =\operatorname{shrink}\left(\frac{\beta_{k}^{2}}{\mu}+\nabla u_{k}, \frac{\phi_{i}^{\prime}(|d|)}{\mu}\right),
\end{aligned}
$$

where the shrink operator $\operatorname{shrink}(a, \theta)$ is defined [30] as:

$$
\operatorname{shrink}(a, \theta)=\max \left(\|a\|_{1}-\theta, 0\right) \frac{a}{\|a\|_{1}},
$$
follow:

Last, the $u$-subproblem is a least square problem as

$$
u_{k+1}=\min _{u}\|B u+b\|_{2}^{2},
$$

where $b=\left(z_{k+1}-\beta_{k}^{1} / \mu, d_{k+1}-\beta_{k}^{2} / \mu\right)$. So the solution $u_{k+1}$ of the least square problem (22) equivalent to the solution of the $B^{T} B u=-B^{T} b$. That is to say, the solution is as follows:

$$
\left(I+\nabla^{T} \nabla\right) u=z_{k+1}-\frac{\beta_{k}^{1}}{\mu}+\nabla^{T}\left(d_{k+1}-\frac{\beta_{k}^{2}}{\mu}\right)
$$

To sum up, the ADMM algorithm can be summarized in Algorithm 1.

\section{Experimental Results}

In this section, we show five sets of experimental results. Test images include synthetic, natural, and real medical ultrasound images. In addition, the denoising effect of the model is compared with that of the existing models, such as TV model [7], ATV model [5], JIN's model [8], and finite difference for the new model.

For the algorithm 1, the stopping condition is that the solution of two adjacent iterations satisfies:

$$
\frac{\left\|u_{k}-u_{k+1}\right\|_{2}}{\left\|u_{k}\right\|_{2}}<10^{-3} \text { and } k>M
$$

where $M$ is the maximum iteration numbers; $M=100$ for algorithm 1. $u_{k}$ represents the results of the $k t h$ iterations. For the JIN's model and finite difference for the new model, we calculated the noise deviation reduction (NDR) at each iteration as a convergence condition;

$$
N D R=\text { mean } \frac{\left(f-u_{k}\right)}{\sqrt{u_{k}}},
$$

And the stopping condition (NDR) for finite difference for the new model is as follow

$$
|\sqrt{N D R}-\sigma|<10^{-1}
$$




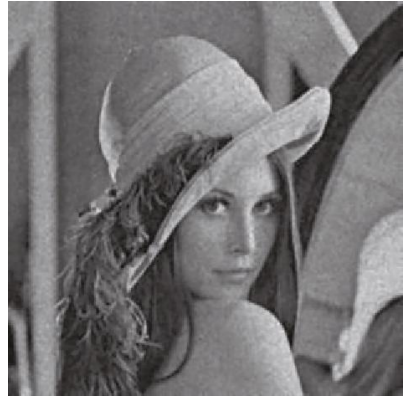

(a)

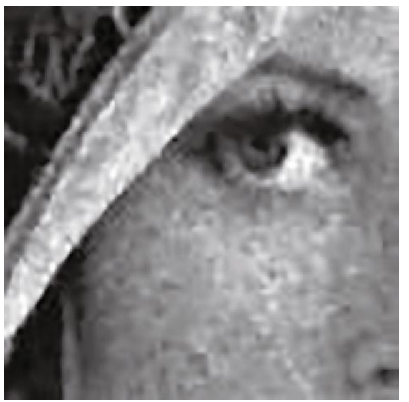

(e)

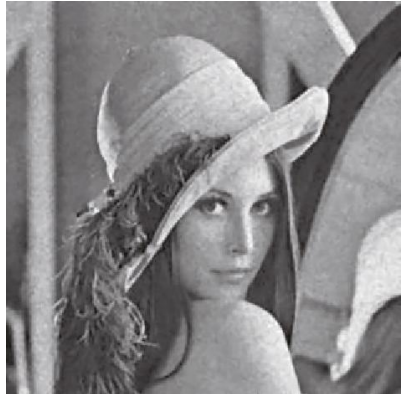

(b)

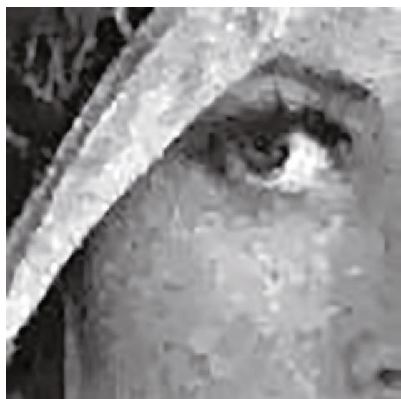

(f)

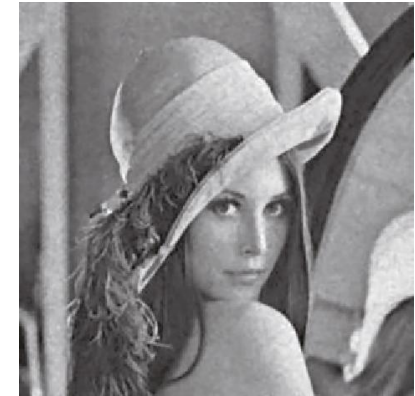

(c)

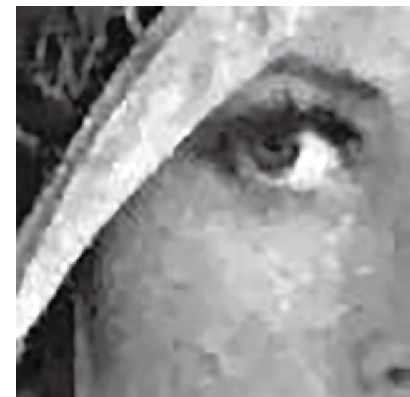

(g)

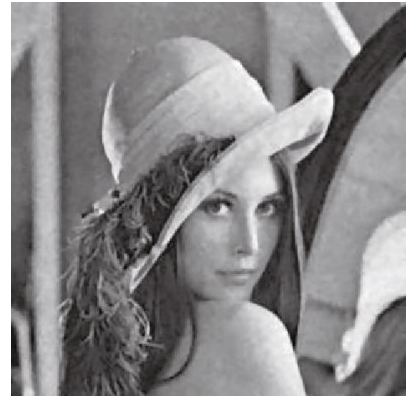

(d)

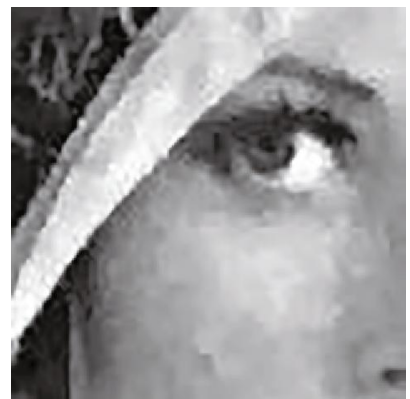

(h)

FIgURE 9: Numerical result of the "lena" image with noise standard deviation $\sigma=2$. (a) Restored image by the TV model. (b) Restored image by the JIN's mode. (c) Restored image by finite difference for the new model. (d) Restored image by ADMM for the new model. (e-h) About the detailed image of $(\mathrm{a}-\mathrm{d})$, respectively.

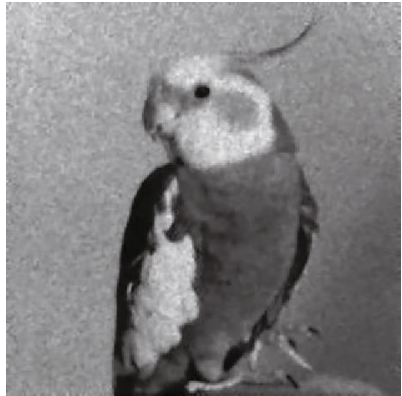

(a)

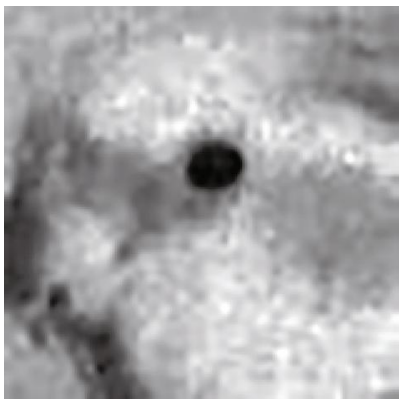

(e)

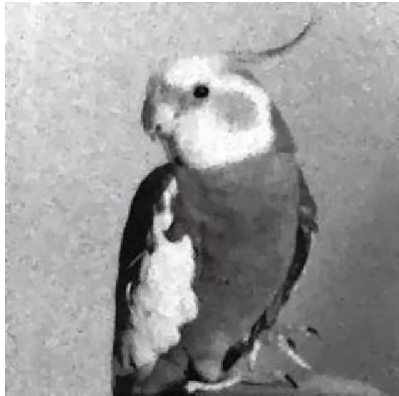

(b)

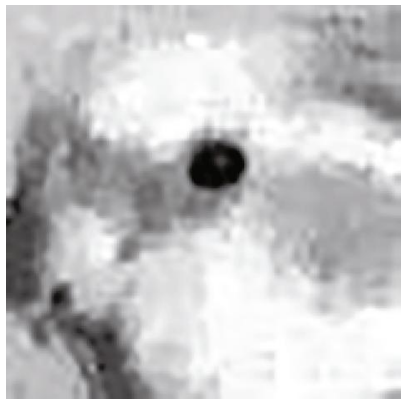

(f)

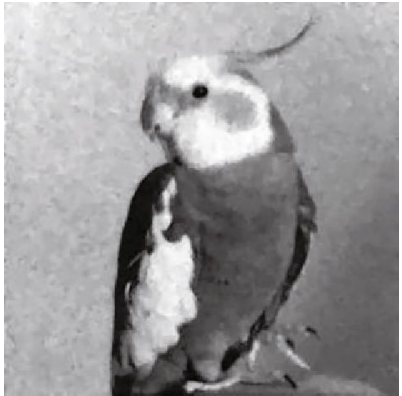

(c)

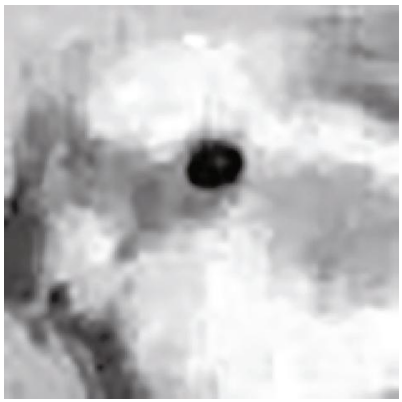

(g)

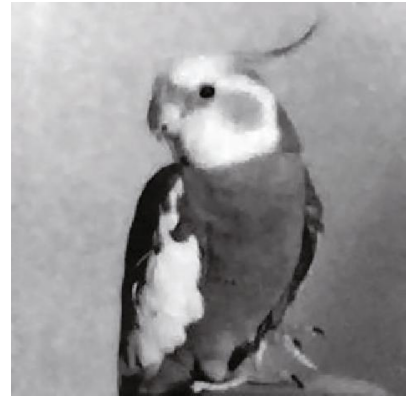

(d)

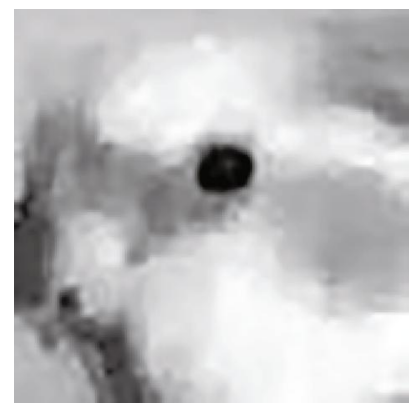

(h)

FIGURE 10: Numerical result of the "bird" image with noise standard deviation $\sigma=2$. (a) Restored image by the TV model. (b) Restored image by the JIN's mode. (c) Restored image by finite difference for the new model. (d) Restored image by ADMM for the new model. (e-h) About the detailed image of $(\mathrm{a}-\mathrm{d})$, respectively. 


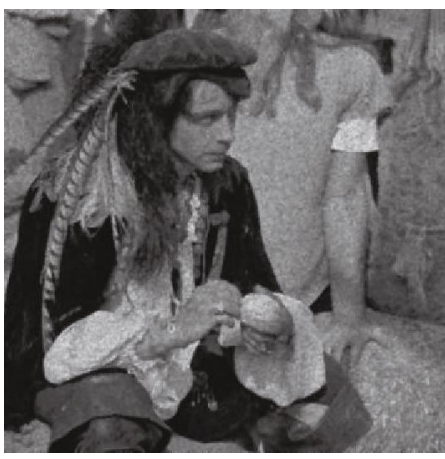

(a)

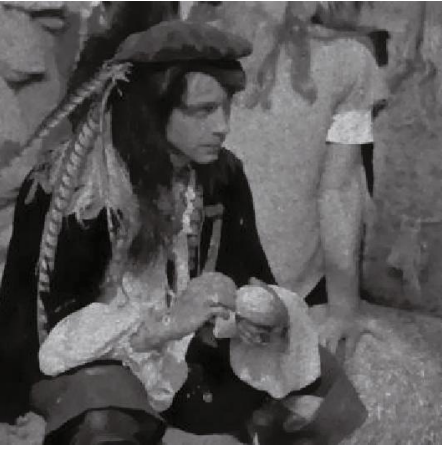

(b)

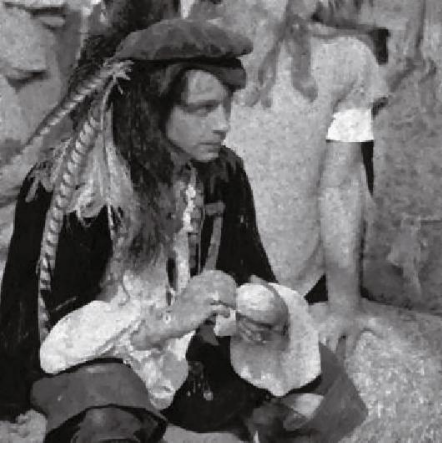

(c)

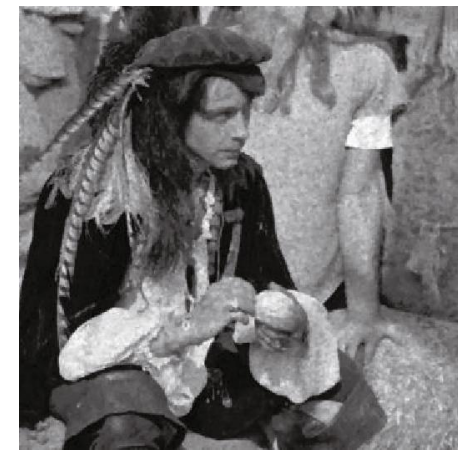

(d)

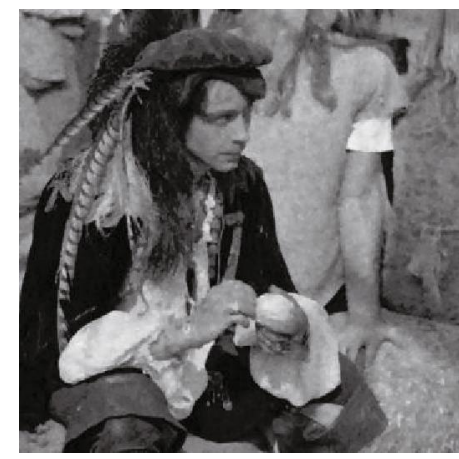

(e)

Figure 11: Numerical result of the "pirate" image with noise standard deviation $\sigma=3$. (a) Restored image by the TV model; (b) Restored image by the ATV model; (c) Restored image by the JIN's model; (d) Restored image by finite difference for the new model; (e) Restored image by ADMM for the new model.

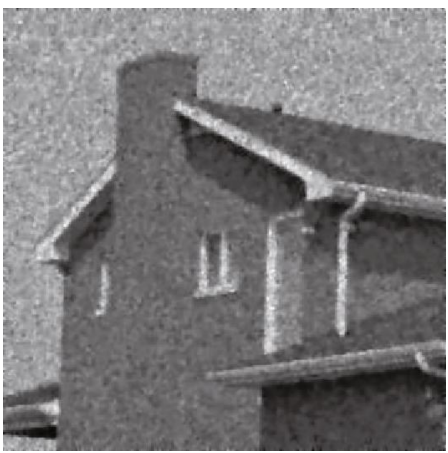

(a)

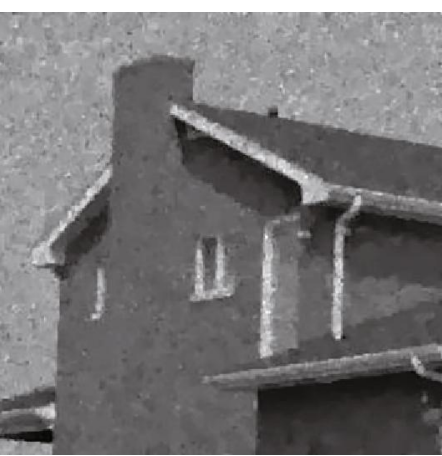

(b)

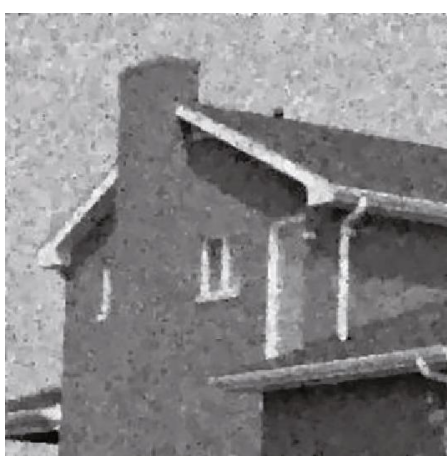

(c)

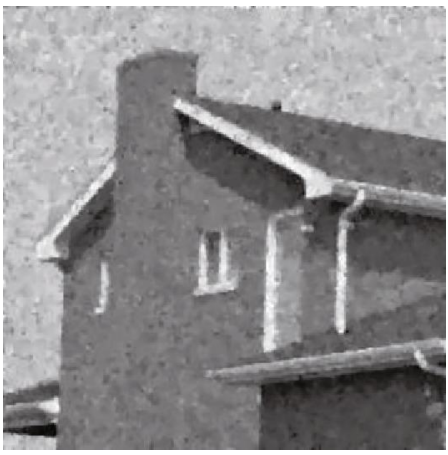

(d)

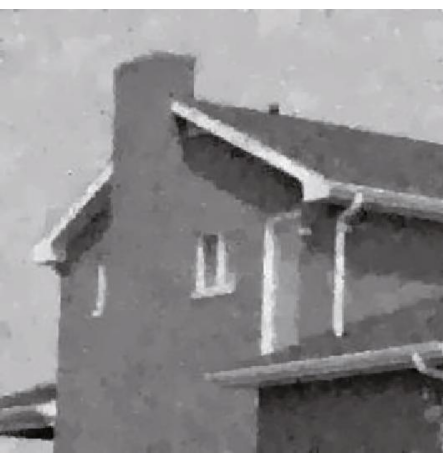

(e)

FIgURE 12: Numerical result of the "house" image with noise standard deviation $\sigma=3$. (a) Restored image by the TV model. (b) Restored image by the ATV model. (c) Restored image by the JIN's model. (d) Restored image by finite difference for the new model. (e) Restored image by ADMM for the new model. 


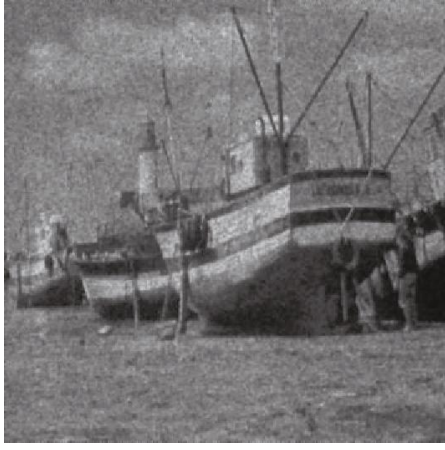

(a)

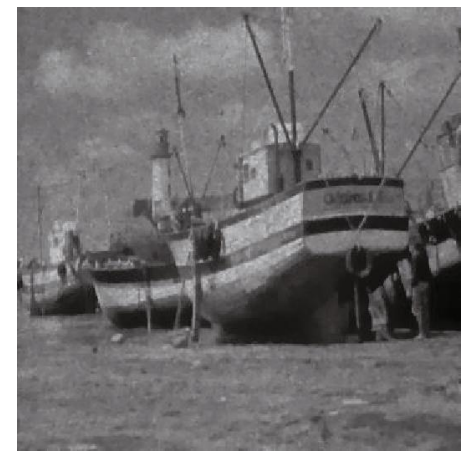

(b)

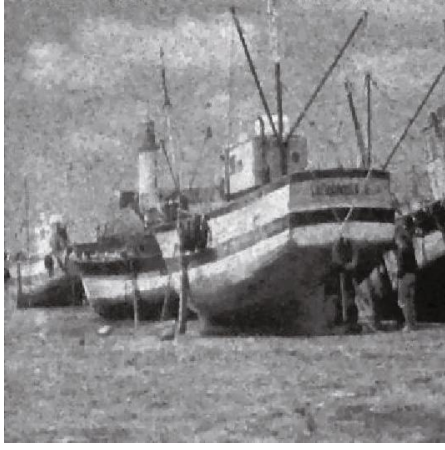

(c)

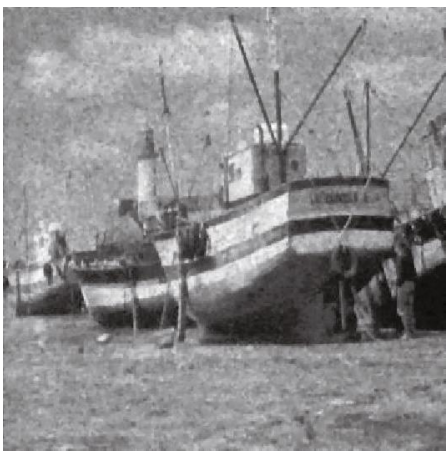

(d)

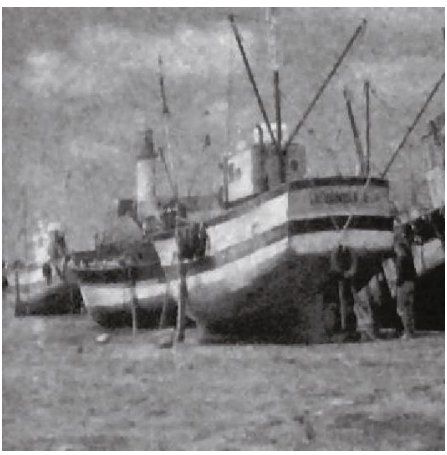

(e)

FIGURE 13: Numerical result of the "boat" image with noise standard deviation $\sigma=4$. (a) Restored image by the TV model. (b) Restored image by the ATV model. (c) Restored image by the JIN's model. (d) Restored image by finite difference for the new model. (e) Restored image by ADMM for the new model.

The effect of image denoising can be evaluated from two aspects. The first is the subjective aspect: it is judged by the subjective consciousness of peoples. The second is the objective aspect: it evaluates the image denoising situation through scientific indicators. In this paper, we evaluate the effect of image denoising by calculating the peak signal-to-noise ratio (PSNR) and structural similarity, which are defined as follows:

$$
\begin{aligned}
& \operatorname{PSNR}(u, \bar{u})=10 \log _{10} \frac{255^{2} m n}{\|u-\bar{u}\|_{2}^{2}}, \\
& \operatorname{SSIM}(u, \bar{u})=\frac{\left(2 \mu_{u} \mu_{\bar{u}}+c_{1}\right)\left(2 \sigma_{\bar{u}}+c_{2}\right)}{\left(\mu_{u}{ }^{2}+\mu_{\bar{u}}{ }^{2}+c_{1}\right)\left(\sigma_{u}{ }^{2}+\sigma_{\bar{u}}{ }^{2}+c_{2}\right)},
\end{aligned}
$$

where $u \in R^{m \times n}$ is the clean image and $\bar{u} \in R^{m \times n}$ is the restored image. $\mu_{u}$ and $\mu_{\bar{u}}$ are the mean intensity of $u$ and $\bar{u}$, respectively. $\sigma_{u}$ and $\sigma_{\bar{u}}$ are the standard deviation of $u$ and $\bar{u}$, respectively. $\sigma_{u \bar{u}}$ is the covariance of $u$ and $\bar{u}$, and $c_{1}$ and $c_{2}$ are some constants for stability.

Best denoising performance are given in bold.

4.1. The Effect of Different Parameters of the Model on Denoising. In the first experiment, we find the optimal parameters $\delta$ value in the algorithm 1 for original image in Figure 1. We choose "syn1" and the noise level is $\sigma$ $=2$. Figure 2 shows that numerical experiments with dif- ferent parameters $\delta$ in algorithm 1. Figure 3 shows that the different PSNR and SSIM values when different $\delta$ values are used in algorithm 1. Here, we can see that the optimal values of PSNR and SSIM values are at $\delta=$ 50. Therefore, in the following experiment, we choose $\delta$ $=50$ in algorithm 1 .

4.2. Denoising Effect of the ADMM Algorithm. In the second experiment, we mainly evaluate the denoising effect of the ADMM algorithm by testing image "syn1" and "syn2". Figures 4(a) and 5(a) show the noise images, and the noise deviation are $\sigma=2$ and $\sigma=3$, respectively. Figures $4(\mathrm{~b})$ and $5(\mathrm{~b})$ show the restored image by the JIN's model. Figures 4(c) and 5(c) show the restored image by finite difference for the new model. Figures 4(d) and 5(d) show the restored image by ADMM for the new model. In addition, Figure 6 shows that the different PSNR and SSIM values when used different models and algorithm. In Figure 6, we can find that our new algorithm has better denoising effect than JIN model and finite difference for the new model.

4.3. Comparison with TV Model, ATV Model, JIN's Model, and Finite Difference for the New Model. In the third experiment, we compare the denoising effects of the TV model, ATV model, JIN's model, and finite difference for the new model with ADMM for the new model by 


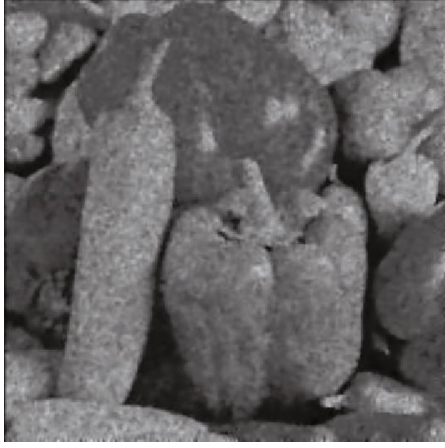

(a)

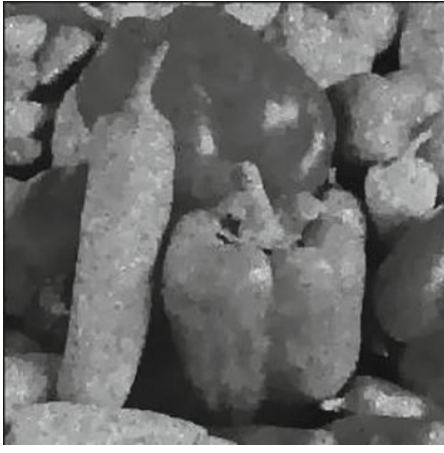

(b)

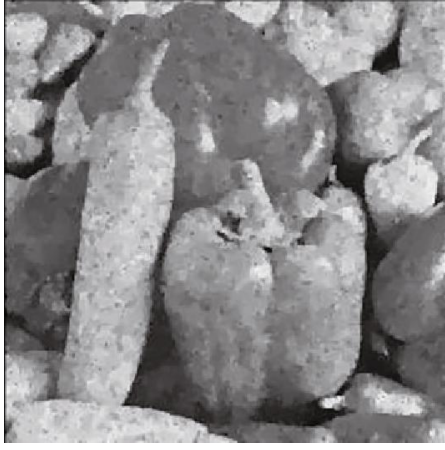

(c)

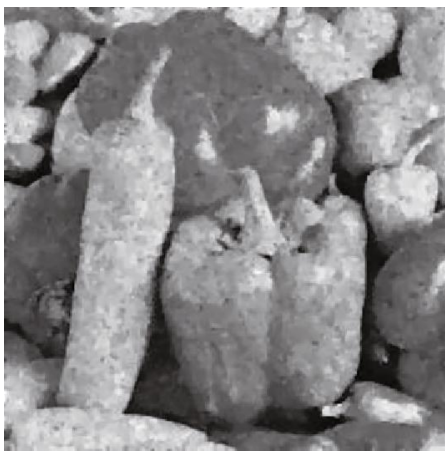

(d)

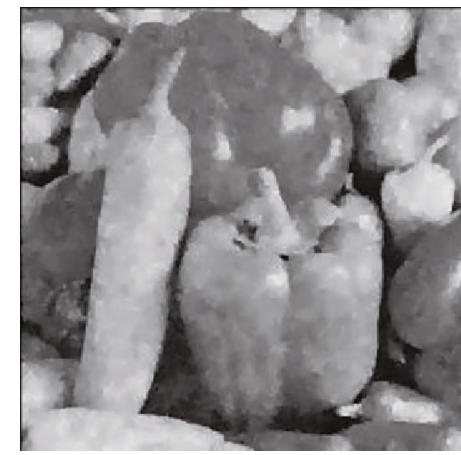

(e)

FIgURE 14: Numerical result of the "peppers" image with noise standard deviation $\sigma=4$. (a) Restored image by the TV model. (b) Restored image by the ATV model. (c) Restored image by the JIN's model. (d) Restored image by finite difference for the new model. (e) Restored image by ADMM for the new model.

TABLE 1: The PSNR of the restored images by the different model.

\begin{tabular}{lcccccc}
\hline Image & $\sigma$ & $\begin{array}{c}\text { TV } \\
\text { (PSNR/SSIM) }\end{array}$ & $\begin{array}{c}\text { ATV } \\
(\text { PSNR/SSIM) }\end{array}$ & $\begin{array}{c}\text { JIN's } \\
\text { (PSNR/SSIM) }\end{array}$ & $\begin{array}{c}\text { Finite difference for the new model } \\
(\text { PSNR/SSIM) }\end{array}$ & $\begin{array}{c}\text { ADMM for the new model } \\
(\text { PSNR/SSIM) }\end{array}$ \\
\hline Lena & 2 & $29.49 / 0.8306$ & $29.94 / 0.8731$ & $29.98 / 0.8665$ & $30.65 / 0.8831$ & $\mathbf{3 0 . 8 3 / 0 . 9 0 4 2}$ \\
Bird & 2 & $28.99 / 0.6907$ & $30.28 / 0.7849$ & $30.36 / 0.7774$ & $31.07 / 0.8173$ & $\mathbf{3 1 . 7 4 / 0 . 8 7 4 7}$ \\
Pirate & 2 & $28.33 / 0.8975$ & $27.22 / 0.8530$ & $28.51 / 0.9042$ & $28.74 / 0.9060$ & $\mathbf{2 8 . 8 1 / 0 . 9 1 5 3}$ \\
House & 2 & $27.45 / 0.6185$ & $29.06 / 0.8101$ & $28.77 / 0.6876$ & $29.74 / 0.7447$ & $\mathbf{3 0 . 3 5 / 0 . 8 1 5 7}$ \\
Boat & 2 & $27.26 / 0.8240$ & $27.90 / 0.8574$ & $28.35 / 0.8622$ & $28.64 / 0.8661$ & $\mathbf{2 8 . 8 1 / 0 . 8 8 0 8}$ \\
Peppers & 2 & $27.60 / 0.7183$ & $28.66 / 0.8377$ & $29.46 / 0.8195$ & $29.53 / 0.8274$ & $\mathbf{2 9 . 6 4 / 0 . 8 4 3 0}$ \\
Lena & 3 & $27.73 / 0.7609$ & $27.98 / 0.8149$ & $28.49 / 0.8298$ & $28.75 / 0.8266$ & $\mathbf{2 8 . 8 5 / 0 . 8 7 1 7}$ \\
Bird & 3 & $27.36 / 0.6451$ & $27.92 / 0.6930$ & $28.48 / 0.7307$ & $26.13 / 0.7701$ & $\mathbf{2 9 . 7 3 / 0 . 8 4 5 6}$ \\
Pirate & 3 & $26.35 / 0.8392$ & $26.38 / 0.8332$ & $26.81 / 0.8578$ & $27.82 / 0.6896$ & $\mathbf{2 7 . 0 5 / 0 . 8 7 2 0}$ \\
House & 3 & $26.08 / 0.5752$ & $27.33 / 0.6611$ & $27.24 / 0.6551$ & $26.82 / 0.7989$ & $\mathbf{2 8 . 2 5 / 0 . 7 8 4 6}$ \\
Boat & 3 & $25.79 / 0.7481$ & $26.47 / 0.7986$ & $26.68 / 0.8029$ & $27.37 / 0.7516$ & $\mathbf{2 7 . 0 3 / 0 . 8 2 1 8}$ \\
Peppers & 3 & $25.93 / 0.6758$ & $26.70 / 0.7409$ & $27.13 / 0.7446$ & $27.19 / 0.7641$ & $\mathbf{2 7 . 5 7 / 0 . 8 0 0 4}$ \\
Lena & 4 & $26.17 / 0.6925$ & $26.94 / 0.8129$ & $26.82 / 0.7625$ & $27.51 / 0.7282$ & $\mathbf{2 7 . 2 5 / 0 . 8 1 9 4}$ \\
Bird & 4 & $25.66 / 0.5679$ & $26.49 / 0.6584$ & $27.25 / 0.7263$ & $25.77 / 0.8159$ & $\mathbf{2 7 . 6 3 / 0 . 7 9 9 4}$ \\
Pirate & 4 & $24.03 / 0.7652$ & $24.33 / 0.7205$ & $24.75 / 0.7787$ & $26.43 / 0.6437$ & $\mathbf{2 5 . 7 9 / 0 . 8 3 0 6}$ \\
House & 4 & $24.63 / 0.5130$ & $25.30 / 0.5760$ & $26.18 / 0.6399$ & $25.51 / 0.7388$ & $\mathbf{2 6 . 5 1 / 0 . 7 3 8 5}$ \\
Boat & 4 & $24.47 / 0.6807$ & $24.95 / 0.7338$ & $25.24 / 0.7370$ & $25.83 / 0.7011$ & $\mathbf{2 5 . 6 1 / 0 . 7 7 4 5}$ \\
Peppers & 4 & $24.49 / 0.6153$ & $25.59 / 0.7153$ & $25.63 / 0.6800$ & & $\mathbf{2 6 . 1 9 / 0 . 7 6 0 0}$ \\
\hline
\end{tabular}

subjective and objective evaluation criteria. The test original images are shown in Figure 7 ("lena," "pirate," "boat," "bird," "house," and "peppers").
Firstly, in the subjective evaluation, we mainly focus on the details of the restored images. The first line of Figure 8 shows the original images ("lena" and "bird") and the noise 


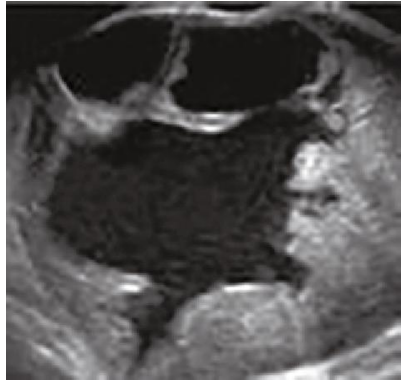

(a)

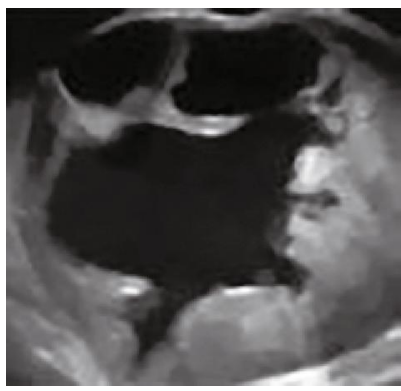

(c)

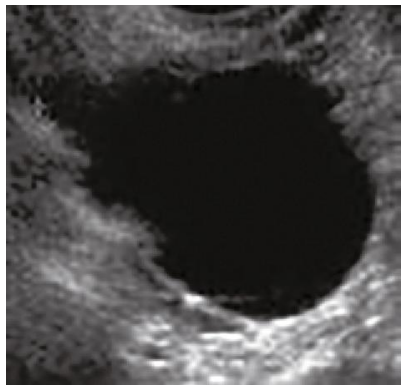

(e)

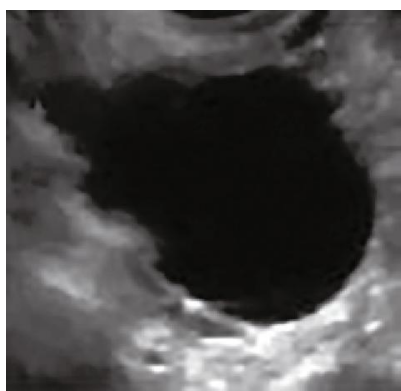

(g)

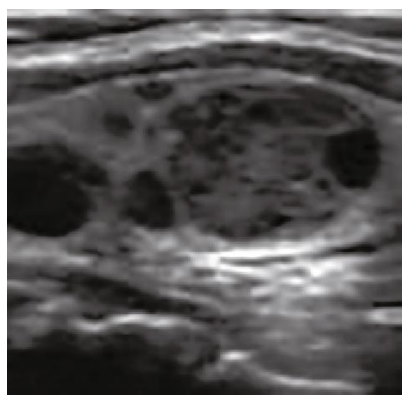

(i)

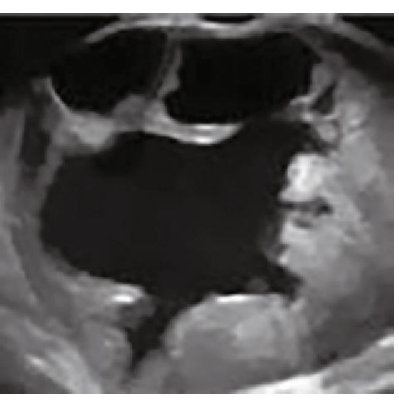

(b)

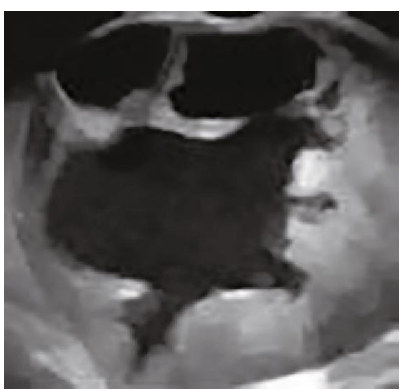

(d)

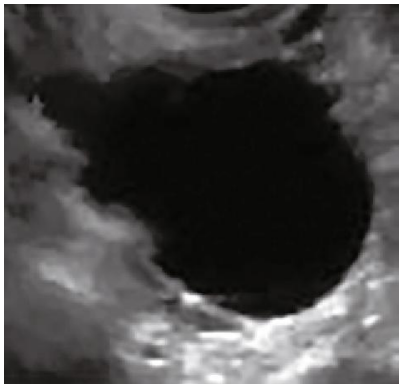

(f)

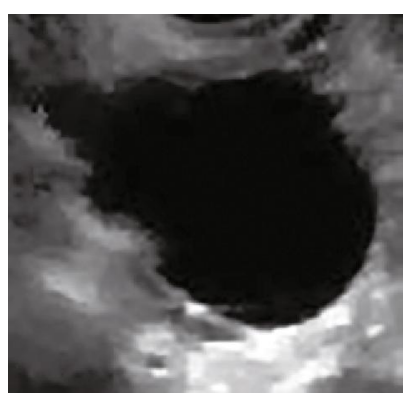

(h)

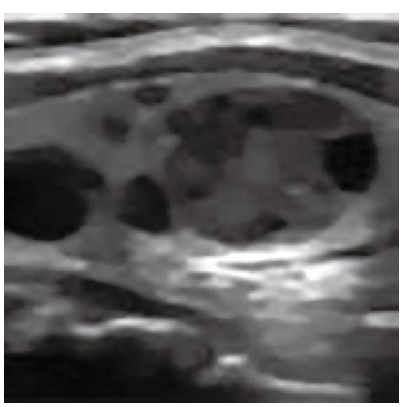

(j)

Figure 15: Continued. 


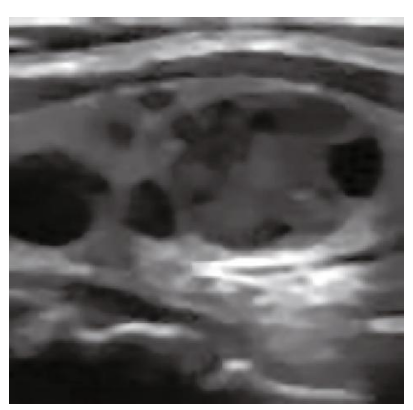

$(\mathrm{k})$

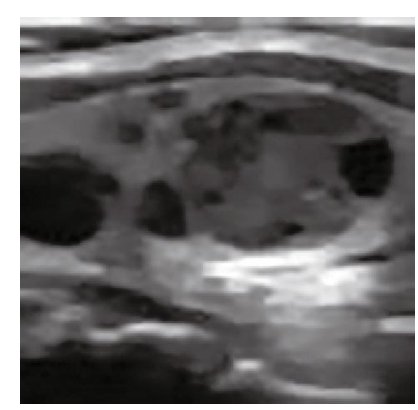

(1)

Figure 15: Numerical result of the real ultrasound image. (a, e, i) Real ultrasound images. (b, f, j) Restored image by the JIN's model. (c, g, k) Restored image by finite difference for the new model. ( $\mathrm{d}, \mathrm{h}, \mathrm{l})$ Restored image by ADMM for the new model.

images with noise deviation is $\sigma=2$. The second line is the corresponding detail images in the first line. Figures 9 and 10 show the restoration results of the noisy images ("lena" and "bird"). Figures 9(a), 9(d), 10(a), and 10(d) were restored images of the TV model, JIN's model, finite difference for the new model, and ADMM for the new model, respectively. Figures 9(e), 9(h), 10(e), and 10(h) are corresponding details images of Figures 9(a), 9(d), $10(\mathrm{a})$, and $10(\mathrm{~d})$, respectively. By observing at the detailed images in Figures 9 and 10, we can see that the denoising effects of the four models are different. Figures 9(e), 9(f), 10(e), and 10(f)show that the denoising effect of TV model JIN's model are worse in subjective vision, and Figures $9(\mathrm{~g}), 9(\mathrm{~h})$, $10(\mathrm{~g})$, and $10(\mathrm{~h})$ show that the denoising effect of finite difference for the new model and ADMM for the new model are well in subjective vision. In addition, we can clearly see the staircase effect in the details images obtained by the TV model and JIN's model. At the same time, by comparing with finite difference for the new model, the staircase effect of the new model is greatly reduced after it is repaired by the ADMM algorithm. This means that using the ADMM algorithm to solve the new energy minimization model has a better effect than other model algorithms in removing speckle noise.

Secondly, in the objective evaluation, we compare the denoising effects of different models with PSNR and SSIM values. Figures 11, 12, 13, and 14(a) shows the restoration results for images through TV model; Figures 11, 12, 13, and 14(b) shows the restoration results for images through ATV model; Figures 11, 12, 13, and 14(c) show the restoration results for images through JIN's model; Figures 11, 12, 13 and 14(d) show the restoration results for images through finite difference for the new model; Figures 11, 12, 13, and 14(e) show the restoration results for images through ADMM for the new model. The noise versions of "boat" and "house" and "pirate" and "bird" are obtained by degradation model (1) with standard deviation 3 and 4, respectively. Table 1 shows the PSNR and SSIM values for different test images by using the TV model, ATV model, JIN's model, finite difference for the new model, and ADMM for the new model. From these values, we observe that the denoising effect of the ADMM for the new model is better than other models and finite difference for the new model.
4.4. Denoising of Real Ultrasound Images. The last experiment is mainly to test the denoising effect of the new algorithm for solving the real ultrasound image and compare it with other denoising models. However, the real ultrasound image does not have the original image, so it cannot be evaluated using the original PSNR and SSIM. In 2005, Buades et al. [31] proposed an evaluation method without original image. The method evaluates the image denoising effect based on the difference and the estimated noise images between the real ultrasound image and the restored image. The difference image and the estimated noise image are defined as:

$$
f-\bar{u} \text { and } \frac{f-\bar{u}}{\sqrt{\bar{u}}}
$$

where $f \in R^{m \times n}$ is the real ultrasound image and $\bar{u} \in R^{m \times n}$ is the restored image. The image denoising effect can be evaluated based on the difference image and the similarity of the estimated noise image, that is, based on the residual image (the difference between the difference image and estimated noise image). If the texture of the residual image is small, the denoising effect is well.

Figure 15 shows that the experimental results of real ultrasound images by applying JIN's model, finite difference for the new model, and ADMM for the new model. Figure 16 shows the residual image after applying the JIN model, finite difference for the new model, and ADMM for the new model to restore the real ultrasound image. Visually speaking, the new model has fewer textures of residual images under the new algorithm, which shows that our method is better in denoising effect.

\section{Conclusion}

In this paper, we introduce the ADMM algorithm to optimize the denoising effect of the model for the new speckle noise recovery model based on adaptive variational method. The new algorithm is a computational framework for solving optimization problems, which is suitable for solving distributed convex optimization problems. The numerical experiments results show the effectiveness of 


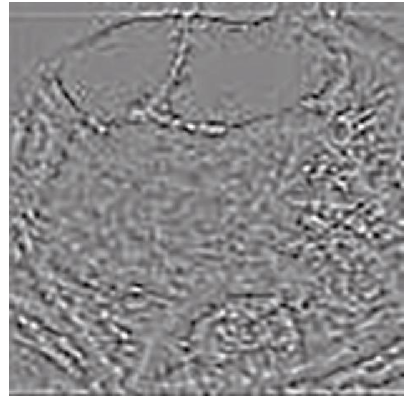

(a)

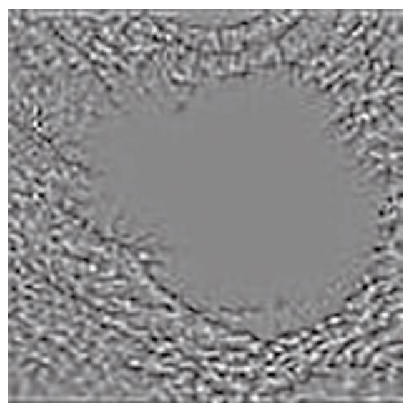

(d)

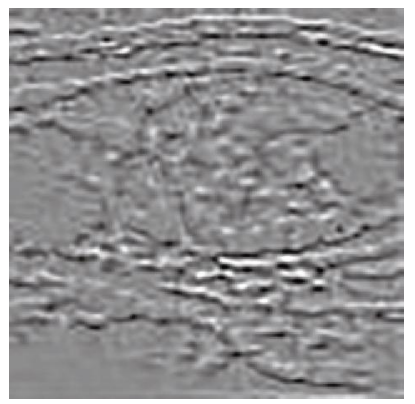

(g)

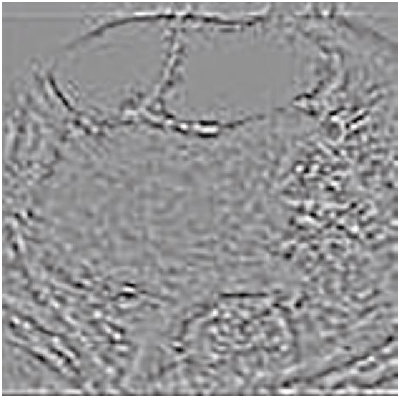

(b)

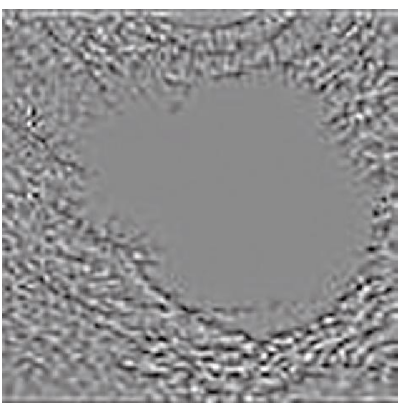

(e)

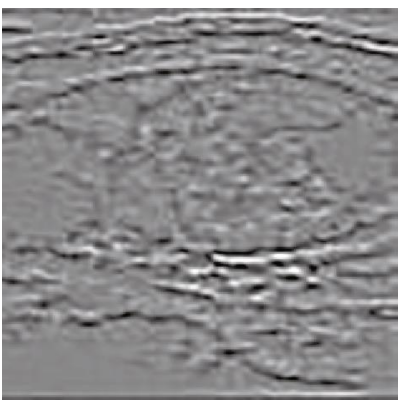

(h)

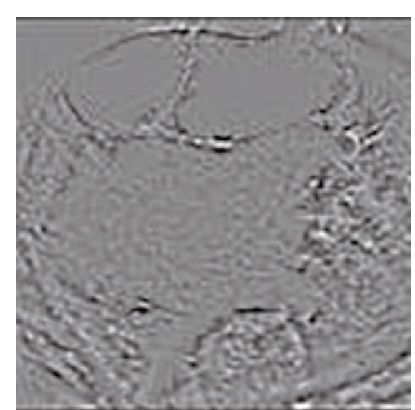

(c)

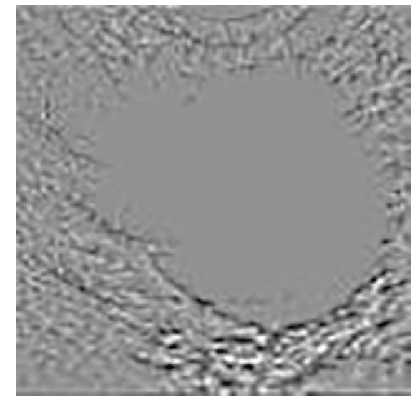

(f)

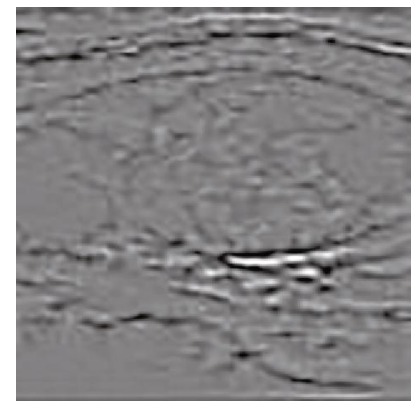

(i)

Figure 16: Residual image. (a, d, g) Residual image by the JIN's model. (b, e, h) Residual image by finite difference for the new model. (c, f, i) Residual image by ADMM for the new model.

the new method. In addition, by comparing with some existing methods, the experiment results show the high efficiency of the new method in image restoration. In recent years, nonconvex model denoising methods have received more and more attention, so it is possible to study nonconvex models in the next work.

\section{Appendix}

\section{A. The Proof of Theorem 3.1.1}

Proof. Firstly, the function $\phi(s)$ is convex, according to Definition 2.1.2, for any $u_{1}, u_{2} \in \Omega$, and $t \in[0,1]$, we have

$\int_{\Omega} \varphi\left(\left|\nabla\left(t u_{1}+(1-t) u_{2}\right)\right|\right) \mathrm{d} x \leq t \int_{\Omega} \varphi\left(\left|\nabla u_{1}\right|\right) \mathrm{d} x+(1-t) \int_{\Omega} \varphi\left(\left|\nabla u_{2}\right|\right) \mathrm{d} x$ meanwhile, we have

$$
\begin{aligned}
\int_{\Omega} & \frac{\left(f-\left(t u_{1}+(1-t) u_{2}\right)\right)^{2}}{t u_{1}+(1-t) u_{2}} \mathrm{~d} x \\
& =\int_{\Omega}\left(\frac{f^{2}}{t u_{1}+(1-t) u_{2}}-2 f+t u_{1}+(1-t) u_{2}\right) \mathrm{d} x \\
& \leq \int_{\Omega}\left(\frac{f^{2}\left(t u_{2}+(1-t) u_{1}\right)\left(t u_{1}+(1-t) u_{2}\right)}{\left(t u_{1}+(1-t) u_{2}\right) u_{1} u_{2}}-2 f+t u_{1}+(1-t) u_{2}\right) \mathrm{d} x \\
& =\int_{\Omega}\left(\frac{f^{2}\left(t u_{2}+(1-t) u_{1}\right)}{u_{1} u_{2}}-2 f+t u_{1}+(1-t) u_{2}\right) \mathrm{d} x \\
& =\int_{\Omega}\left(\frac{t f^{2}}{u_{1}}-2 t f+t u_{1}+\frac{(1-t) f^{2}}{u_{2}}-2(1-t) f+(1-t) u_{2}\right) \mathrm{d} x \\
& =\int_{\Omega} t \frac{\left(f-u_{1}\right)^{2}}{u_{1}}+(1-t) \frac{\left(f-u_{2}\right)^{2}}{u_{2}} \mathrm{~d} x,
\end{aligned}
$$


Therefore,

$$
E\left(t u_{1}+(1-t) u_{2}\right) \leq t E\left(u_{1}\right)+(1-t) E\left(u_{2}\right) .
$$

This proof is established.

\section{B. The Proof of Theorem 3.1.2}

Proof. According to Theorem 3.1.1, we have

$$
\begin{aligned}
E\left(\frac{u_{1}+u_{2}}{2}\right)= & \int_{\Omega} \varphi\left(\left|\nabla\left(\frac{1}{2} u_{1}+\frac{1}{2} u_{2}\right)\right|\right) \mathrm{d} x+\alpha \int_{\Omega} \frac{\left(f-\left(u_{1}+u_{2} / 2\right)\right)^{2}}{u_{1}+u_{2} / 2} \mathrm{~d} x \\
\leq & \frac{1}{2} \int_{\Omega} \varphi\left(\left|\nabla u_{1}\right|\right) \mathrm{d} x+\frac{1}{2} \int_{\Omega} \varphi\left(\left|\nabla u_{2}\right|\right) \mathrm{d} x+\alpha \int_{\Omega} \\
& \cdot\left(\frac{f^{2}\left(1 / 4\left(u_{1}+u_{2}\right)^{2}-1 / 4\left(u_{1}-u_{2}\right)^{2}\right)}{\left(u_{1}+u_{2} / 2\right) u_{1} u_{2}}-2 f+\frac{1}{2} u_{1}+\frac{1}{2} u_{2}\right) \mathrm{d} x \\
\leq & \frac{1}{2} \int_{\Omega} \varphi\left(\left|\nabla u_{1}\right|\right) \mathrm{d} x+\frac{1}{2} \int_{\Omega} \varphi\left(\left|\nabla u_{2}\right|\right) \mathrm{d} x \\
& +\alpha \int_{\Omega}\left(\frac{\left(f-u_{1}\right)^{2}}{2 u_{1}}+\frac{\left(f-u_{2}\right)^{2}}{2 u_{2}}-\frac{f^{2}\left(u_{1}-u_{2}\right)^{2}}{2\left(u_{1}+u_{2}\right) u_{1} u_{2}}\right) \mathrm{d} x \\
= & \frac{1}{2} E\left(u_{1}\right)+\frac{1}{2} E\left(u_{2}\right)-\alpha \int_{\Omega} \frac{f^{2}\left(u_{1}-u_{2}\right)^{2}}{2\left(u_{1}+u_{2}\right) u_{1} u_{2}} \mathrm{~d} x \\
= & E\left(u_{1}\right)-\alpha \int_{\Omega} \frac{f^{2}\left(u_{1}-u_{2}\right)^{2}}{2\left(u_{1}+u_{2}\right) u_{1} u_{2}} \mathrm{~d} x
\end{aligned}
$$

If $u_{1} \neq u_{2}$, then the above assumption give a contradiction that $u_{1}$ is not a minimize solution.

\section{The Proof of Cubic Equation (24) Only One Positive Real Number Solution}

Proof. For the cubic equation, it has three solutions. Now, we assume $\left(z^{1}, z^{2}, z^{3}\right)$ are solution of cubic equation (24). According to Vieta theorem, we have

$$
\begin{aligned}
z^{1}+z^{2}+z^{3} & =-\frac{\alpha-2 \beta_{k}^{1}-2 \mu u_{k}}{2 \mu} \\
z^{1} z^{2}+z^{1} z^{3}+z^{2} z^{3} & =0 \\
z^{1} z^{2} z^{3} & =\frac{\alpha f^{2}}{2 \mu}>0
\end{aligned}
$$

It is easy to know that the cubic equation has at least one real solution and $z^{i} \neq 0$ for all $i=1,2,3$. Now, let us analyze the three solutions, without loss of generality, we assume $z^{1}+z^{2}=\xi, z^{1} z^{1}=\rho$, and $z^{3}$ are a real solution.

The first case: assume $z^{3}$ is positive. So we can know $z^{1} z^{2}=$ $\rho>0$ and $z^{1}+z^{2}=\xi<0$. Meanwhile, $\left(z^{1}, z^{2}\right)$ are solution of equation $z^{2}-\xi z+\rho=0$; thus, $z^{1}=\xi+\sqrt{\xi^{2}-4 \rho} / 2$ and $z^{2}=$ $\xi-\sqrt{\xi^{2}-4 \rho / 2}$.

If $\xi^{2}-4 \rho<0$, then $z^{1}$ and $z^{2}$ are complex numbers. If $\xi^{2}-4 \rho \geq 0$, then $\xi \pm \sqrt{\xi^{2}-4 \rho} / 2<0$. Thus, $z^{1}$ and $z^{2}$ are negative.
The second case: assume $z^{3}$ is negative. So we can know $z^{1} z^{2}=\rho<0$ and $z^{1}+z^{2}=\xi<0$. Meanwhile, $\left(z^{1}, z^{2}\right)$ are solution of equation $z^{2}-\xi z+\rho=0$; thus, $z^{1}=\xi+\sqrt{\xi^{2}-4 \rho} / 2$ and $z^{2}=\xi-\sqrt{\xi^{2}-4 \rho} / 2$.

Because of $\xi^{2}-4 \rho>\xi^{2}>0$, therefore, $z^{1}$ and $z^{2}$ are real number, $z^{1}$ is positive, and $z^{2}$ is negative.

This proof is established.

\section{Data Availability}

The experimental data are obtained by MATLAB R2017a, $2.93 \mathrm{GHz}$ cup, $4 \mathrm{G}$ ram, and windows 7 .

\section{Conflicts of Interest}

The authors declare that they have no conflicts of interest.

\section{Authors' Contributions}

All authors typed, read, and approved the final manuscript.

\section{Acknowledgments}

This paper is partially supported by the Natural Science Foundation of Guangdong Province (2018A030313364), the Special Innovation Projects of Universities in Guangdong Province (2018KTSCX197), the Science and Technology Planning Project of Shenzhen City (JCYJ20180305125609379), and the China Scholarship Council Project (201508440370).

\section{References}

[1] J.-S. Lee, "Speckle suppression and analysis for synthetic aperture radar images," Optical Engineering, vol. 25, no. 5, article 255636, 1986.

[2] K. Krissian, R. Kikinis, and C. Westin, "Speckle-constrained filtering of ultrasound images," in IEEE Computer Society Conference on Computer Vision and Pattern Recognition (CVPR'05), pp. 547-552, San Diego, CA, USA, 2005.

[3] P. G. Hou, H. F. Gu, and Y. T. Wang, "Research of image denoising based on wavelet threshold," Applied Mechanics and Materials, vol. 109, pp. 690-694, 2011.

[4] W. Zhang and G. Song, "Signal denoising in wavelet domain based on a new kind of thresholding function," Journal of Xidian University, vol. 2, pp. 296-299, 2004.

[5] K. Krissian, C.-F. Westin, R. Kikinis, and K. G. Vosburgh, "Oriented speckle reducing anisotropic diffusion," IEEE Transactions on Image Processing, vol. 16, no. 5, pp. 14121424, 2007.

[6] M. Kang, M. Kang, and M. Jung, "Total generalized variation based Denoising models for ultrasound images," Journal of Scientific Computing, vol. 72, no. 1, pp. 172-197, 2017.

[7] L. I. Rudin, S. Osher, and E. Fatemi, "Nonlinear total variation based noise removal algorithms," Physica D Nonlinear Phenomena, vol. 60, no. 1-4, pp. 259-268, 1992.

[8] Z. Jin and X. Yang, "A Variational model to remove the multiplicative noise in Ultrasound Images," Journal of Mathematical Imaging and Vision, vol. 39, no. 1, pp. 62-74, 2011. 
[9] T. Loupas, W. N. McDicken, and P. L. Allan, "An adaptive weighted median filter for speckle suppression in medical ultrasonic images," IEEE Transactions on Circuits and Systems, vol. 36, no. 1, pp. 129-135, 1989.

[10] Z. Zong and F. Hu, "Lpsolutions of infinite time interval backward doubly stochastic differential equations," Univerzitet $u$ Nišu, vol. 31, no. 7, pp. 1855-1868, 2017.

[11] Z. Zong and F. Hu, "_L_- ${ }^{-P_{-}}$solutions of infinite time interval backward doubly stochastic differential equations under monotonicity and general increasing conditions," Journal of Mathematical Analysis and Applications, vol. 458, no. 2, pp. 1486-1511, 2018.

[12] F. Hu and Z. Chen, " $L^{p}$ solutions of anticipated backward stochastic differential equations under monotonicity and general increasing conditions," Stochastics: An International Journal of Probability and Stochastic Processes, vol. 88, no. 2, pp. 267284, 2016.

[13] H. Wu, Y. Ren, and F. Hu, "Continuous dependence property of BSDE with constraints," Applied Mathematics Letters, vol. 45, pp. 41-46, 2015.

[14] F. Hu, "Dynamically consistent nonlinear evaluations with their generating functions in L p," Acta Mathematica SinicaEnglish Series, vol. 29, no. 4, pp. 815-832, 2013.

[15] X. Zhang, L. Liu, and Y. Wu, "Multiple positive solutions of a singular fractional differential equation with negatively perturbed term," Mathematical and Computer Modelling, vol. 55, no. 3-4, pp. 1263-1274, 2012.

[16] X. Zhang, Y. Wu, and L. Caccetta, "Nonlocal fractional order differential equations with changing-sign singular perturbation," Applied Mathematical Modelling, vol. 39, no. 21, pp. 6543-16552, 2015.

[17] X. Zhang, L. Liu, Y. Wu, and Y. Cui, "Existence of infinitely solutions for a modified nonlinear Schrodinger equation via dual approach," Electronic Journal of Differential Equations, vol. 2147, pp. 1-15, 2018.

[18] X. Zhang, L. Liu, Y. Wu, and Y. Cui, “The existence and nonexistence of entire large solutions for a quasilinear Schrodinger elliptic system by dual approach," Journal of Mathematical Analysis and Applications, vol. 464, no. 2, pp. 1089-1106, 2018.

[19] X. Zhang, L. Liu, Y. Wu, and Y. Cui, "Existence and asymptotic properties of solutions for a nonlinear Schrodinger elliptic equation from geophysical fluid flows," Applied Mathematics Letters, vol. 90, pp. 229-237, 2019.

[20] X. Zhang, J. Xu, J. Jiang, Y. Wu, and Y. Cui, “The convergence analysis and uniqueness of blow-up solutions for a Dirichlet problem of the general k-Hessian equations," Applied Mathematics Letters, vol. 102, pp. 106-124, 2020.

[21] B. Chen, S. Huang, Z. Liang, W. Chen, and B. Pan, "A fractional order derivative based active contour model for inhomogeneous image segmentation," Applied Mathematical Modelling, vol. 65, no. 1, pp. 120-136, 2019.

[22] B. Chen, Q.-H. Zou, W.-S. Chen, and Y. Li, "A Fast Regionbased Segmentation Model with Gaussian Kernel of Fractional Order," Advances in Mathematical Physics, vol. 2013, Article ID 501628, 7 pages, 2013.

[23] B. Chen and W.-S. Chen, "Noisy image segmentation based on wavelet transform and active contour model," Applicable Analysis, vol. 90, no. 8, pp. 1243-1255, 2010.

[24] B. Chen, Q. Zou, and Y. Li, “A new image segmentation model with local statistical characters based on variance minimiza- tion," Applied Mathematical Modelling, vol. 39, no. 12, pp. 3227-3235, 2015.

[25] P. L. Lions and B. Mercier, "Splitting algorithms for the sum of two nonlinear operators," SIAM Journal on Applied Mathematics, vol. 16, no. 6, pp. 964-979, 1979.

[26] J. Eckstein and D. P. Bertsekas, "On the Douglas-Rachford splitting method and the proximal point algorithm for maximal monotone operators," Mathematical Programming, vol. 55, no. 1-3, pp. 293-318, 1992.

[27] R. T. Rockafellar, "Minimax theorems and conjugate saddlefunctions," Mathematica Scandinavica, vol. 14, pp. 151-173, 1964.

[28] T. Goldstein and S. Osher, "The split Bregman method for L1regularized problems," SIAM Journal on Imaging Sciences, vol. 2, no. 2, pp. 323-343, 2009.

[29] W. Deng and W. Yin, "On the global and linear convergence of the generalized alternating direction method of multipliers," Journal of Scientific Computing, vol. 66, no. 3, pp. 889-916, 2016.

[30] Y. L. Wang, J. F. Yang, and W. T. Yin, "A new alternating minimization algorithm for total variation image reconstruction," SIAM Journal of Scientific Computing, vol. 1, no. 3, pp. 248272, 2008.

[31] A. Buades, B. Coll, and J. M. Morel, "A non-local algorithm for image denoising," IEEE Computer Society Conference on Computer Vision and Pattern Recognition, vol. 2, pp. 60-65, 2005. 